Revista de Psicología Vol. 39 (2), 2021 (e-ISSN 2223-3733)

\title{
Factores psicosociales asociados a la participación política no convencional en una muestra de jóvenes ciudadanos en Lima, Perú
}

\author{
Meir Álvaro Tintaya Orihuela ${ }^{1}$, Rosa María Luisa Martina Cueto Saldívar ${ }^{2}$ \\ Pontificia Universidad Católica del Perú-Perú
}

En el contexto local, la participación política (PP) juvenil ha sido limitada a su ámbito representativo, y los jóvenes han sido catalogados como apáticos políticos y desinteresados de su compromiso cívico con la sociedad, sin indagar por otras modalidades de PP. Por ello, desde el modelo psicosocial, se realiza un primer estudio (1) que explora los repertorios de PP, así como sus atribuciones y motivaciones de participación; en caso del segundo estudio, (2) se analiza las relaciones entre las variables socio-cognitivas (Conocimiento Político, Interés en Política y Eficacia Política), actitudinales (Confianza Política y Cinismo Políticos) y emocionales vinculadas a la PP no convencional en una muestra de jóvenes en Lima. En el primer estudio, se realizó 8 entrevistas a jóvenes afiliados a organizaciones civiles, cuyas respuestas fueron analizadas en base al enfoque de análisis temático. En base a los resultados cualitativos, se identifica (1) la concepción de PP, (2) los motivos de PP, y (3) los riesgos y limitantes de la PP. En caso del segundo estudio, participaron 132 jóvenes en Lima a través de encuestas online; posteriormente, realizó se realizaron análisis correlacionales, de regresión múltiple y path analysis. En base a los resultados, se identifican dos modalidades de PP: contenciosas y de expresión cívica, y se evidencia un modelo explicativo para la PP de expresión cívica, en el cual el Cinismo Político, la Eficacia Política y el Interés en Política tiene efecto sobre la PP. Se discute la importancia de los resultados para explicar el involucramiento de los jóvenes limeños en la política.

Palabras clave: Psicología Política, Participación Política, Interés en Política, Eficacia Política, Ciudadanía

1 Licenciado en Psicología Social y magíster en Psicología. Asistente de docencia e investigador de Opinión Pública en la Pontificia Universidad Católica del Perú, Perú. Dirección postal: Ca. Manuel Toribio Ureta 221, La Victoria. COD: 15034. Contacto: mtintaya@pucp.pe https://orcid.org/0000-0002-6359-7372

2 Licenciada en Psicología Social, magíster en Psicología Comunitaria y doctora en Psicología. Profesora principal en la Pontificia Universidad Católica del Perú. Dirección postal: Avenida Ricardo Palma 828 Departamento 702 San Antonio Miraflores, Contacto: rcueto@pucp.pe https://orcid.org/0000-0003-3549-2001 


\section{Psychosocial factors associated with unconventional political participation in a sample of young citizens in Lima, Peru}

Young Political Participation (PP) has been limited to a representative domain, and young Peruvians have been cataloged as politically disinterested and apathetic with their civic commitment to society, without inquiring about other types of political participation. Thus, from a psychosocial approach, two studies were conducted: the first seeks to (1) explore the typology, attributions and motivations of PP; (2) the second study analyzes the relationships between socio-cognitive factors (Political Knowledge, Political Interest, and Political Efficacy), attitudes (Political Trust and Political Cynism), and emotions linked to non-conventional PP in a sample of 18- to-25-year-old people of Lima, Peru. In the first study, eight interviews were conducted with young people affiliated to civil organizations, whose responses were analyzed from a thematic analysis approach. From these qualitative results, three main themes were identified: (1) a conception of political participation, (2) the motives for political participation, (3) the risks and limitations of political participation. In case of the second research, 132 youths in Lima participated in an online survey, which was submitted to correlational analyses, multiple regressions and path analysis. These results suggest two types of non-conventional PP: an contentious PP and civic-expressive PP, the latter of which is explained by political cynism and political efficacy, mediated by political interest. Implications and importance of results are discussed in relation to human development, political involvement and construction of citizenship among young Peruvians.

Keyswords: political psychology, political participation, political interest, political efficacy, citizenship

\section{Fatores psicossociais associados à participaçáo política náo convencional em uma amostra de jovens cidadáos de Lima, Peru}

No contexto local, a participaçáo política juvenil (PP) tem se limitado à sua esfera representativa, e os jovens são classificados como políticos apáticos e desinteressados em seu compromisso cívico com a sociedade, sem questionar outras formas de PP. Portanto, a partir do modelo psicossocial, é realizado um primeiro estudo (1) que explora os repertórios da PP, bem como suas atribuiçóes e motivaçóes para a participação; No caso do segundo estudo, (2) as relaçōes entre as variáveis sociocognitivas (Conhecimento Político, Interesse em Política e Eficácia Política), atitudinais (Confiança Política e Cinismo Político) e variáveis emocionais ligadas à PP náo convencional são analisadas em um amostra de jovens em Lima. No primeiro estudo, foram realizadas 8 entrevistas com jovens filiados a organizaçóes civis, cujas respostas foram analisadas com base no enfoque da análise temática. Com base nos resultados qualitativos, identificamos (1) a concepção da PP, (2) os motivos da PP e (3) os riscos e limitaçôes da PP. No caso do segundo estudo, 132 jovens de Lima participaram de pesquisas online, posteriormente, foram realizadas análises correlacionais, regressóes múltiplas e análises de caminhos. A partir dos resultados, identificam-se duas modalidades de PP: contenciosa e de expressão cívica, e evidencia-se um modelo explicativo para o PP de expressão cívica, em que o Cinismo Político, a Eficácia Política e o Interesse pela Política afetam o PP. Discute-se a importância dos resultados para explicar o envolvimento dos jovens de Lima na política.

Palavras-chave: Psicologia Política, Participação Política, Interesse pela Política, Eficácia Política, Cidadania 
Facteurs psychosociaux associés à la participation politique non conventionnelle dans un échantillon de jeunes citoyens à Lima, Pérou

Dans le contexte local, la participation politique des jeunes (PP) a été limitée à leur sphère représentative, et les jeunes ont été classés comme des politiciens apathiques et désintéressés par leur engagement civique envers la société, sans enquêter sur d'autres formes de PP. Ainsi, à partir du modèle psychosocial, une première étude est menée (1) qui explore les répertoires des PP, ainsi que leurs attributions et motivations de participation; Dans le cas de la seconde étude, (2) les relations entre les variables socio-cognitives (connaissances politiques, intérêt pour la politique et efficacité politique), attitudinales (confiance politique et cynisme politique) et les variables émotionnelles liées à la PP non conventionnelle sont analysées dans un échantillon de jeunes à Lima. Dans la première étude, 8 entretiens ont été menés avec des jeunes affiliés à des organisations civiles, dont les réponses ont été analysées sur la base de l'approche d'analyse thématique. Sur la base des résultats qualitatifs, nous identifions (1) la conception du PP, (2) les raisons du PP, et (3) les risques et les limites du PP. Dans le cas de la deuxième étude, 132 jeunes de Lima ont participé à des enquêtes en ligne, puis des analyses corrélationnelles, des régressions multiples et des analyses de chemin ont été effectuées. Sur la base des résultats, deux modalités de PP sont identifiées: contentieuse et d'expression civique, et un modèle explicatif pour le PP d'expression civique est mis en évidence, dans lequel le cynisme politique, l'efficacité politique et l'intérêt pour la politique ont un effet sur le PP. L'importance des résultats pour expliquer l'implication des jeunes de Lima dans la politique est discutée.

Mots clés: psychologie politique, participation politique, intérêt pour la politique, efficacité politique, citoyenneté 
La participación política (PP) está sujeta a las coyunturas sociales y políticas, y es una temática que responde al modo en que las personas se involucran como sujetos políticos dentro de la sociedad. Desde una aproximación psicosocial, su análisis considera desde el estudio de la tipología o modos de expresión política hasta la comprensión de aquello que motiva a las personas a participar políticamente (Sorribas $\&$ Brussino, 2017).

La base de la PP está en la interacción entre las personas y el sistema político, ya sea individual o colectivamente (Valencia, 1990; van Deth, 2014). En ese contexto, la PP se entiende como el conjunto de acciones, legales o no, que tienen como objetivo influir directa o indirectamente sobre el sistema político, resaltando su cualidad instrumental (Barnes \& Kasse, 1979). Para Conge (1988), mínimamente la PP debe incluir las formas activas de acción de naturaleza intencional, alude a la condición de ciudadanía de los involucrados y, hace referencia al gobierno y a la política en general. Debido a que la gestión de los servicios públicos (salud, educación, justica, vivienda, entre otras) tiene efecto en la calidad de vida de las personas, la PP se orienta a influir en la selección de los representantes políticos, sus decisiones, y la estructura del sistema político mismo (Sabucedo, 1998; Torcal, Moreno \& Toerell, 2006).

La conceptualización de la PP se ha orientado preferentemente hacia los procesos electorales y de activismo partidario, cuya expresión máxima sigue siendo el voto. Este previo delineamiento se suele denominar PP convencional, la cual se fundamenta en la noción de democracia representativa, donde el sistema político institucional define las oportunidades, lineamientos y modos de participación en política (Barnes \& Kasse, 1979; Valencia, 1990). Diversos estudios tipifican las acciones específicas de la PP convencional, tales como asistir a mítines, apoyar económicamente a un partido o candidato, votar, y persuadir a otros para que opten por la propia preferencia partidaria 
(Delfino \& Zubieta, 2010; Sorribas \& Brussino, 2017). Sin embargo, la PP no puede orientarse únicamente al voto como apoyo o rechazo de candidatos y sus propuestas, pues sería restringir los matices y alcances de otras formas de involucrarse como actores políticos dentro de la sociedad.

Cuando la participación se distancia de los canales tradicionales representativos, se alude a una PP no convencional (Barnes \& Kasse, 1979; Sabucedo, 1988; Torcal et al., 2006). Este tipo de PP debe ser entendida como el conjunto de acciones directas que se despliegan en el espacio público, con el objetivo de obtener resultados políticos o visibilizar un posicionamiento ideológico dentro de la sociedad (van Deth, 2001). La PP no convencional se enmarca en la noción de democracia participativa; es decir, los propios ciudadanos fomentan sus modos de hacer política y tratan de redistribuir el poder a favor de la sociedad. Específicamente, su repertorio incluye la firma de peticiones, escritos a la prensa, asociacionismo, boicots, protestas, huelgas y manifestaciones; incluso, considera el cierre de calles, ocupar edificios y fábricas, el agravio personal y el daño de propiedades. Estas últimas formas de PP tiene matices con la violencia y la ilegalidad; sin embargo, el criterio de legalidad no puede ser condicionante de la PP no convencional, pues una acción catalogada como ilegal o legal varía según el contexto histórico, cultural y político de cada país (Delfino \& Zubieta, 2014). Dado el incremento en el uso de tecnologías de redes sociales, también se consideran las discusiones en foros sobre temas públicos, así como el publicar y comentar enlaces con contenido político como parte del espectro no convencional (Gil de Zúniga \& Valenzuela, 2012; Gil de Zúńiga, Molyneux \& Zheng, 2014; Lilleker \& Koc-Michalska, 2017).

Si bien aún se mantiene una mirada dualista de la PP, en la última década, los estudios empiezan a optar por una perspectiva multidimensional de la PP. Así, si se considera el grado de irrupción en el espacio público, se puede diferenciar entre participación pacífica y agresiva (Delfino, Zubieta \& Muratori, 2013), o entre organizada, normativa y no normativa (Sandoval \& Hatibovic, 2018). Si se contempla los objetivos de demanda de la PP, por un lado, se identifica la participa- 
ción orientada hacia los responsables políticos que se ajusta a normas auto-expresivas de ciudadanía vigilante (Sorribas \& Brussino, 2017). Por otro lado, la participación orientada hacia la sociedad y el sistema político implica experiencias participativas en asociaciones cívicas vinculadas al compromiso social, con objetivos ambientalistas, feministas o de defensa de derechos (Botero, Torres \& Alvarado, 2008). Cabe decir que las modalidades de PP no convencional no serían necesariamente excluyentes entre sí, sino que responden a distintos canales de expresión e incidencia política (Mannarini, Legittimo \& Talo, 2008), así como a claves contextuales que moderan las acciones políticas según la agenda política internacional, nacional y las demandas de la ciudadanía (Vilas, Alzate \& Sabucedo, 2016; van Stekelenburg, Klandermans \& van Dijk, 2009). En ese sentido, los modos pacíficos de PP no convencional pueden constituirse como indicadores de compromiso cívico, pues permiten expresar los intereses ciudadanos alejados de la política tradicional, usualmente, a través de movimientos sociales (Ocampo, 2011; Rodríguez et al., 1993). No obstante, algunos repertorios de PP no convencional no siempre se mantienen pacíficos, pues dentro del marco de tensión entre la ciudadanía y el Estado, pueden tornarse violentos ante la represión de parte de las autoridades hacia manifestantes en marchas y protestas, o ante agotamiento de los canales de diálogo, desatando reacciones violentas de ambos lados (van Deth, 2001; Sorribas \& Brussino, 2017).

Desde una aproximación psicosocial, lo que motiva a las personas a elegir este tipo de participación política puede involucrar variables instrumentales (Klandermas, 1984), sociopsicológicas (Brussino, Rabbia \& Sorribas, 2009; Delfino \& Zubieta, 2014; Sorribas \& Brussino, 2017), identitarias (Klandermans, 2014; Simon \& Klandermans, 2001; Sabucedo, Durán \& Alzete, 2010), emocionales (Jasper, 2011; Sabucedo \& Vilas, 2014, Sandoval \& Da Silva, 2016) y deontológicas (Sabucedo, Dono, Alzate \& Seoane, 2018; van Stekelenburg, 2013; Vilas \& Sabucedo, 2012). Incluso, a nivel colectivo, el modelo teórico de conciencia política (Sandoval, 2001) integra factores psicosociales afines a los previamente mencionados. 
De manera general, la propuesta teórica de conciencia política (Sandoval, 1994, 2001; Sandoval \& Da Silva, 2016) considera que existen dimensiones psicosociales que constituyen un estado de conciencia individual sobre la sociedad y sobre sí mismo como miembro de la sociedad, de modo que proveen de significado e información sobre el funcionamiento de la sociedad para que las personas decidan involucrarse o no como actores políticos.

\section{Factores psicosociales asociados a la participación política no convencional}

La disposición de actuar políticamente, de manera convencional o no convencional, se basa en una visión personal sobre el medio social, compuesta por creencias y expectativas sobre la cultura política hegemónica y las relaciones dentro de la sociedad, dando cabida a la propia opinión sobre la situación actual (Berreota \& Sandoval, 2014; Botero et al., 2008; Sandoval \& Da Silva, 2016). Así, la participación de las personas como actores políticos depende del grado de conciencia sobre dichas creencias y expectativas sociales; y puede variar desde el sentido común, que asume como natural las posiciones inequitativas de los grupos sociales y las relaciones establecidas entre ellos, hasta la conciencia crítica que cuestiona las condiciones de la vida cotidiana. Para ello, desde el marco socio-cognitivo, la PP puede ser analizada desde procesos actitudinales, estructuras cognitivas y afectivas sobre el funcionamiento de la sociedad y el sistema político (John, Fieldhouse \& Liu, 2011).

Diversos estudios indican que el (1) conocimiento político, (2) el interés en la política, (3) la confianza política, (4) el cinismo político, (5) la eficacia política y (6) la identidad colectiva (Brussino, Rabbia \& Sorribas, 2009; Delfino \& Zubieta, 2014; Mannarini, Roccato, Fedi \& Rovere, 2009; Sorribas \& Brussino, 2017; van Zomeren et al., 2008; Sabucedo, Durán \& Alzate, 2010) inciden en la PP no convencional.

En el caso del conocimiento político, este se define como el grado de entendimiento que una persona tiene sobre la dinámica política en la cual se encuentra inmerso (Krampen, 2000), y que dependen del 
nivel educativo, edad, consumo de medios de comunicación y del grado de deliberación política (Sergovia, 2016). No obstante, los niveles de conocimiento político suelen ser bajos en varios países y no necesariamente son factores determinantes de la PP (Somin, 2016). Como estructura cognitiva, el conocimiento político por sí solo resulta incompleto para explicar por qué las personas se involucran como agentes políticos, pues la PP depende de bases motivacionales como el interés en política (Brussino, Medrano, Sorribas \& Rabbia, 2011; Reichert, 2018). Esta última consiste en la sensación de curiosidad por los asuntos públicos, así la información política captada como saliente ayuda a las personas a configurar la percepción sobre el mundo político y a seguir buscando información relacionada (Luskin, 1990). Esta combinación de conocimiento e interés en política permitiría sedimentar nodos y redes asociativas sobre conceptos políticos (Miller, 2011), facilitando la toma de conciencia de los temas de agenda pública, la historia detrás de los sucesos políticos y sociales, las coyunturas más relevantes, las posturas partidarias; incluso, anticipar las posibles consecuencias de las decisiones gubernamentales (Grönlund \& Milner, 2006).

Siguiendo con el rol motivacional de la PP, el vínculo con algún objeto, estructura o proceso del sistema político también puede valorarse subjetivamente en términos de confianza política. Esta refiere al grado de seguridad sobre el funcionamiento del sistema político, incluyendo las decisiones del gobierno, la capacidad de gestión de las instituciones públicas, y los resultados obtenidos (Eisinger, 2000; Hardin, 2000; Hooghe \& Zmerli, 2013). En América Latina, la confianza institucional es baja en democracias consolidas, mientras que es alta en países con regímenes autoritarios (Edelman, 2019). Pese a esta situación, la confianza se puede construir cuando las instituciones públicas ejercen un trato igualitario, cumplen sus promesas, son fiscalizadas y admiten sus errores en política; siendo contingente al crecimiento del PBI y a la valoración de la situación económica nacional (Bargsted \& Torcal, 2013; Latinobarómetro, 2017).

Cuando la evaluación de las instituciones públicas es afectada por la crisis económica y política, emerge el cinismo político como factor 
disuasivo de la PP. No solo se trata de una sensación de desesperanza sobre la política en general, sino en una aversión hacia la clase política, y la percepción de que la política es un sistema estancado (Pattyn, van Heil, Dhont \& Onraet, 2012) Esta actitud política parte de un cuestionamiento de los motivos reales de los políticos cuando asumen cargos públicos, y suele nutrirse de la percepción de un sistema normativo débil e incapaz de promover el orden social (Beramendi \& Zubieta, 2013); más aún, cuando la corrupción y la ineficiencia de los gobiernos aparece como males endémicos dentro del sistema político. Por tanto, el cinismo político puede ser considerado como un peligro potencial para la democracia, en tanto obstruye los procesos de PP, desalienta la deliberación política, e incrementa las manifestaciones extremas (Eisinger 2000; Opdycke, Segura \& Vásquez, 2013). No obstante, la desconfianza no es lo mismo que cinismo, pues la percepción de que las instituciones no generan los resultados esperados puede derivar en que los propios ciudadanos critiquen el sistema y se movilicen políticamente a través de protestas, reforzando la democracia (Rivera, 2019).

Al igual que la desconfianza en las instituciones, cuando los problemas que afectan la política se atribuyen a aspectos situacionales, la percepción de que uno es capaz de realizar un cambio queda limitada (Sandoval, 2001). Por tanto, la eficacia política resulta importante como variable explicativa y diferenciadora de la PP. Esta se entiende como los sentimientos de que el cambio es posible cuando las personas de manera individual o colectiva se involucran como actores políticos en una situación del mundo político y social (Campbell, Gurin \& Miller, 1954 en Sohl, 2014; Krampen, 2000; Mannarini et al., 2008; Sandoval, 2001). Durante el estudio de este concepto, se tendió a considerar dos formas de eficacia política: interna y externa. La eficacia política interna refiere a la percepción de que uno tiene las competencias suficientes para influir y realizar un impacto en asuntos políticos por sus propios medios. En el caso de la eficacia política externa, esta responde a la creencia de que las instituciones y autoridades van a dar respuesta a las intenciones ciudadanas de influir en los aspectos gubernamentales (Caprara, Vecchione, Capanna \& Mebane, 2009; Sohl, 
2014). La eficacia también existe a nivel grupal o colectiva y refiere a la percepción de que un grupo determinado tiene la capacidad y fuerza suficiente para lograr los cambios deseados; estas creencias de eficacia colectiva terminan siendo más decisiva en repertorios contestarios de PP (van Zomeren et al., 2008).

En adición a la eficacia política, puede considerarse la evaluación instrumental de las condiciones contextuales y organizacionales para decidir involucrarse como actor político en acciones que busquen resarcir demandas ciudadanas insatisfechas (Sandoval, 2001; Sandoval \& Da Silva, 2016). Basándose en una lógica racional, la PP puede evaluarse a partir de sus costos y beneficios, por lo que las personas estarían motivadas a participar según sus expectativas sobre la propia contribución y la de otros, sobre todo, en movilizaciones y protestas que demandan cambios ante experiencias de desigualdad (Klandermans, 1984; 2004).

Junto con la eficacia colectiva, la percepción de injusticia y la identidad colectiva enmarcan un modelo explicativo de la PP colectiva. Cuando los ciudadanos evalúan una situación social como injusta y consideran que el estatus de su grupo ha sido ilegítimamente afectado, existe más probabilidad de participar en repertorios colectivos de PP (van Zomeren et al., 2008). Así, la experiencia afectiva de agravio y la identificación grupal derivan en una identidad social politizada y movilizada que, a través de la PP, busca reparar la injusticia percibida (Sabucedo et al., 2010; van Zomeren et al., 2010; ). Desde esta perspectiva, la percepción de injusticia se traduce en reacciones emocionales que movilizan a las personas desenvolverse como sujetos políticos.

La PP, como cualquier actividad humana, también está motivada por emociones. En general, las emociones son estados mentales con una determinada valencia afectiva y un grado de intensidad, que cumple funciones adaptativas, motivacionales y sociales, con el fin de canalizar apropiadamente las reacciones conductuales (Barrett, Mesquita, Ochsner \& Gross, 2007; Chóliz, 2005). A partir de la evaluación de la situación sociopolítica de la sociedad, emergen procesos emocionales que motivan la acción política, dotan de significado la experiencia par- 
ticipativa de los ciudadanos, y pueden actuar como puente entre la sensibilidad a problemas sociales y la acción colectiva (Jasper, 2011; Sandoval \& Da Silva, 2016; Wlodarczyk et al., 2017).

Tradicionalmente, la ira es la emoción negativa que ha sido aceptada como catalizadora del involucramiento en protestas y surge cuando se percibe que ciertos principios morales han sido violados. A nivel intergrupal, la ira es la respuesta emocional ante la privación percibida en un contexto de inequidad, el cual supone una amenaza para el grupo con el que uno se identifica (van Zomeren et al., 2008).

Si bien la ira es una emoción que energiza a los actores políticos, basada en la percepción de injusticia, no explica necesariamente la persistencia de los ciudadanos de participar en acciones de protesta que no generan resultados esperados a corto plazo. Posiblemente, con el fin de superar los costos de las expectativas no cumplidas (Klandermans, 1984; van Stekelenburg, Klandermans \& van Dijk, 2011), las emociones positivas cobran relevancia cuando la PP (protestas) es vista a largo plazo, tales como la esperanza y el optimismo (Sabucedo \& Vilas, 2014; Vilas et al., 2016). La primera es un sentimiento relacionado con las expectativas de que sucederán cambios favorables a futuro, lo cual proporciona un clima emocional positivo y propicio para tomar acciones a largo plazo y enfrentar posibles obstáculos (Ciarrochi, Parker, Kashdan, Heaven \& Barkus, 2015); mientras que la segunda refiere a las expectativas de obtener resultados positivos. Ambas emociones positivas sugieren que la anticipación del éxito será suficiente motivo de PP (Vilas et al., 2016).

Empíricamente, se ha demostrado que el conocimiento político discrimina entre quienes participan y no participan políticamente (Brussino et al., 2009; Sorribas \& Brussino, 2013a), mientras que el interés en política impulsa a los jóvenes a tomar parte de acciones activistas (Rodríguez, Sabucedo \& Costa, 1993), de pedido de firmas, huelgas, y manifestaciones (Vázquez, Panadero \& Rincón, 2006), o de acciones contenciosas hacia el poder legislativo (Sorribas \& Brussino, 2013a; 2013b; 2017). 
Siguiendo con las formas no convencionales de PP, cuando se experimenta baja confianza política, los ciudadanos optan por acciones más contenciosas (Mannarini et al, 2008), o más auto-organizadas y orientadas al bien común (John, Fieldhouse \& Liu, 2011). Asimismo, se ha evidenciado que el cinismo político reduce las posibilidades de involucrarse en estas formas de PP en el caso de jóvenes universitarios y no universitarios de Perú, Italia, y Estados Unidos (Jorge, 2016; Mannarini et al., 2008; Robinson, 2014).

De igual modo, se ha demostrado que la eficacia política facilita los procesos de intervención de jóvenes y adultos en el espacio público, mediante protestas y movilizaciones sociales (Brussino et al., 2009; Mannarini et al., 2009; Páez, Javaloy, Wlodarczyk, Espelt \& Rimé, 2013; Sorribas \& Brussino, 2013a, 2017). En términos sociocognitivos, la autoeficacia dirigida hacia el dominio político facilita el desarrollo de liderazgo y un sentido de control político que llevaría a intervenir en las decisiones políticas locales, así como organizar y movilizar personas en grupos para fines políticos (Sohl, 2014).

Respecto al rol de las emociones en la PP, la evidencia demuestra efectos en conjunto de la ira y las emociones positivas como desencadenantes motivacionales de actividades de protesta social (Sabucedo \& Vilas, 2014; Rico, Alzate \& Sabucedo, 2017; Wlodarczyk et al., 2017). Empíricamente, la ira no solo influye directamente sobre la intención de participar, sino indirectamente a través de las emociones de esperanza, orgullo y optimismo. De ese modo, la ira muestra su carácter constructivo canalizado por las emociones positivas (Alberici, Milesi, Malfermo, Canfora \& Marzana, 2012), así ambos tipos de emociones no pueden ser entendidas aisladamente (Sabucedo y Vilas, 2014).

Como se ha ido desarrollando, los repertorios conductuales de PP no convencional pueden explicarse desde distintas variables cognitivas, actitudinales y emocionales. Ahora, sería imprudente asumir que dichos patrones comportamentales tienen un carácter universal; por el contrario, resulta más preciso considerar que están vinculados a sucesos históricos, sociales y culturales que permiten explicar la expe- 
riencia participativa según el desarrollo de cada sociedad y generación (Sabucedo, 1988; Sandoval, 1994; 2001; Sorribas \& Brussino, 2017).

Para las generaciones más jóvenes, estas modalidades permiten prácticas participativas más horizontales, y visibilizan sus demandas ciudadanas, pues no encuentran espacio en los modelos tradicionales de hacer política que les generan apatía y desconfianza (Aguilera, 2010; Arias-Cardona \& Alvarado, 2015, DiGrazia, 2014; Sola \& Hernández, 2017). Así, se ha encontrado que suelen involucrarse mayormente en acciones políticas de protesta, movilización y de contención (Defino \& Zubieta, 2014; Delfino et al., 2013, Vázquez et al., 2006). De igual modo, prefieren participar en asociaciones universitarias tales como colectivos no partidarios, gremios con mayor complejidad organizacional y en voluntariados, con el fin de canalizar sus intereses sobre educación de calidad, la no violencia hacia la mujer, la protección del medio ambiente, o la defensa de derechos fundamentales (Botero, Torres \& Alvarado, 2008; Berreota \& Sandoval, 2014; Cubides, Borelli, Unda \& Vázquez, 2015).

Estas formas de PP juvenil responden a trayectorias de socialización y aprendizaje sobre normas, valores, ideologías, y códigos simbólicos de una cultura política específica que se da a lo largo de la vida (Imhoff \& Brussino s2017; Pfaff, 2009). De hecho, la adolescencia tardía y la adultez temprana serían las etapas donde las actitudes hacia la política y la PP suelen cambiar con mayor rapidez y, posteriormente, reafirmarse (Neundorf \& Niemi, 2014). Además, la juventud no solo implica una transición de la dependencia hacia la autonomía, sino una iniciación donde se asumen responsabilidades orientadas a integrarse a la sociedad, usualmente, a través del empleo, la educación y la PP (Venturo, 2001). Por tanto, la PP no convencional resultaría importante para el desarrollo integral de los jóvenes como ciudadanos activos que contribuyan a la reflexión y construcción de una sociedad basada en la democracia. 


\section{Juventud y participación política en el Perú}

En el Perú, las trayectorias de socialización política de los jóvenes han tenido cabida en el espacio universitario, mediante la deliberación y participación política. Desde los años 50, los jóvenes cuestionaron las políticas sobre la educación universitaria y se adhirieron a agrupaciones estudiantiles. Entre los 60s y fines de los 70s, los movimientos sociales y juveniles asumen principalmente una orientación política de izquierda, basada en posturas anti-oligárquicas, percibiendo a la clase social alta como un grupo distante de la realidad social (Rosas, 2009). Sin embargo, estos discursos socialistas en los 80 s, se devalúan y las organizaciones de izquierda empezaron a ser vistas como parte de la irresponsabilidad y corrupción en el mundo político, así la culpa de las desigualdades sociales pasa a las figuras políticas en general. Estas fallas en el sistema político y la crisis económica del momento generaron el escenario propicio para la captación de jóvenes - principalmente de sectores históricamente excluidos y desatendidos por el Estado - por organizaciones extremistas, como el Partido Comunista del Perú Sendero Luminoso. Durante los 90s, la situación política se agrava, debido al régimen dictatorial, la corrupción generalizada y la represión autoritaria por efecto de la violencia política, lo cual generó el debilitamiento de la PP universitaria en general (Rosas, 2009; Venturo, 2001).

En consecuencia, la situación política vivida durante los 80 s y 90 s deja un rezago en los modos en que los jóvenes se involucran en el mundo de la política. Por un lado, deja espacio a las agrupaciones políticas como el Movimiento por la Amnistía y Derechos Fundamentales (MOVADEF) busquen jóvenes simpatizantes de sus posturas políticas, validando modalidades violentas de PP (Gamarra, 2012). Por otro lado, cuando se involucran en cualquier acto de protesta o reclamo por sus derechos, los jóvenes pueden ser estigmatizados, y vistos como instigadores, violentos o "terrucos", dificultando la construcción de una cultura de paz (Jave, Cépeda y Uchuypoma, 2015). De ese modo, los jóvenes en el Perú se encuentran en un contexto histórico donde experimentan el mundo político cada vez más cínico e instrumental (Venturo, 2001), con un clima de desconfianza institucional en todos 
los niveles socioeconómicos, y grupo etario (IOP, 2017), sobre todo, hacia los partidos políticos, el Congreso, y el poder judicial (IPSOS, 2017).

Sin embargo, la previa situación descrita no implica necesariamente una indiferencia de los jóvenes hacia la política ni la falta de compromiso cívico (Ames, 2013; Krauskopf, 2000), sino que evidencia la desconfianza hacia el modo tradicional de hacer política; un sentir que se comparte con casi toda Latinoamérica dado el débil desempeño de las instituciones políticas (Brussino et al., 2009; Del Tronco, 2012; Sandoval, 2012). Lo anterior cobra sentido, pues psicológicamente, los jóvenes en Lima configuran una representación social de la política con correlatos afectivos ambiguos; es decir, para este sector la política implica normas e ideologías para dirigir la sociedad y velar por el bienestar, pero, a su vez, se vincula con el engaño, la corrupción y las ambiciones personales (Cueto, Fourment, Seminario \& Fernández, 2014). En estudios sobre la cultura política, Nureña (2015) concluye que el alejamiento de los jóvenes universitarios de UNMSM de la política institucional se debe a la rigidez estructural del sistema de gobierno que tiende a excluir la participación autónoma y que funciona bajo una lógica clientelar, frustrando los intentos por mejorar la calidad de la educación universitaria; mientras tanto, Chaparro (2018) identifica que un grueso segmento de la sociedad responde a grupos de jóvenes renegados e insatisfechos con la democracia del país.

En ese sentido, los jóvenes evidencian involucrarse en formas no convencionales de PP. Por ejemplo, durante la campaña presidencial del 2016, se presentó una marcha contra la candidatura de Keiko Fujimori, con el eslogan de "No a Keiko", alegando a la memoria de los crímenes de corrupción y de lesa humanidad cometidos durante el gobierno de Alberto Fujimori (La República, mayo 2016). De igual modo, cuando el Congreso de la República anunció una reforma laboral juvenil, los jóvenes en situación de empleo, de colectivos universitario, y comités del SENAJU salieron a las calles a reclamar por la reducción de beneficios salariales y laborales; es más, lograron la derogación de esta reforma (Perú 21, enero 2015). Otro ejemplo de PP juvenil sería el movimiento 
"Ni Una Menos" que continuamente busca colocar la violencia hacia la mujer en la agenda pública, a través campañas de sensibilización y marchas (El Comercio, noviembre 2017). Así, las modalidades no convencionales de PP como el reclamo y la protesta surgen en contextos donde los mecanismos formales resultan ser ineficientes, sumándose la deslegitimación del Estado y sus instituciones, las cuales no logran satisfacer las necesidades de ciertos grupos sociales (Grompone \& Tanaka, 2009).

En base a lo descrito, se plantea dos estudios: el primer estudio tiene como objetivo explorar los repertorios de PP de los jóvenes e identificar sus motivos de participación en la política. Cabe resaltar que este objetivo cobra relevancia dada la falta de una tipología local que precise las formas de PP no convencional como ejercicio de ciudadanía en el contexto nacional; además, se debe considerar el contexto universitario, ya que ofrece oportunidades para la reflexión crítica sobre la realidad social peruana, y la deliberación sobre asuntos públicos (Venturo, 2001; Nureńa, 2015). En caso del segundo estudio, se busca analizar las relaciones entre las variables socio-cognitivas (conocimiento político, interés en política y eficacia política), actitudinales (confianza política y cinismo político) y las emociones relevantes asociadas a la PP no convencional en jóvenes limeños.

A través del segundo estudio, se propone contrastar las siguientes hipótesis:

1) El grado de conocimiento político, los niveles de interés en política, la eficacia política interna y el cinismo político se asociarían directamente con modalidades no convencionales de participación política

2) La confianza política mostraría una asociación inversa con los niveles de participación política no convencional.

3) Las emociones de ira, orgullo, esperanza y optimismo se asociarían directamente entre sí. Además, los niveles de ira mostrarían asociaciones inversas con la participación política no convencional, mientras que las emociones positivas se asociarían directamente. 


\section{Estudio 1}

\section{Método}

Este primer estudio tiene como objetivo identificar los repertorios de participación política (PP) de los jóvenes, los motivos y atribuciones de su involucramiento en cada una de estas acciones. Además, esta fase permite precisar las experiencias participativas relevantes de los jóvenes para la adaptación local del instrumento de PP no convencional.

\section{Participantes}

Los entrevistados fueron 8 jóvenes de 21 a 26 años, residentes en Lima, y miembros en organizaciones civiles en los últimos 12 meses. Seis de los participantes cuentan con experiencia previa en otras organizaciones o colectivos activistas.

Entre los criterios de inclusión, se consideró que sean miembros de alguna organización o colectivo activista, que asuman alguna función dentro de ella, y que no sean miembros de algún partido político. Sus características y afiliación organizacional varían según diversas temáticas de interés juvenil, como se observa en el siguiente cuadro.

\section{Tabla 1}

Caracteristicas de los participantes

\begin{tabular}{|c|c|c|c|c|c|}
\hline Sexo & Edad & $\begin{array}{l}\text { Tiempo en la } \\
\text { organización }\end{array}$ & $\begin{array}{l}\text { Tipo de } \\
\text { organización }\end{array}$ & $\begin{array}{l}\text { Experiencia } \\
\text { previa }\end{array}$ & Ocupación \\
\hline Hombre & 23 & 10 meses & $\begin{array}{l}\text { Representación } \\
\text { estudiantil }\end{array}$ & Sí & Estudiante \\
\hline Hombre & 22 & 3 años & $\begin{array}{l}\text { Género y } \\
\text { diversidad } \\
\text { sexual }\end{array}$ & Sí & Estudiante \\
\hline Mujer & 21 & 10 meses & $\begin{array}{l}\text { Representación } \\
\text { estudiantil }\end{array}$ & No & Estudiante \\
\hline Mujer & 25 & 6 años & $\begin{array}{l}\text { Derechos } \\
\text { Humanos }\end{array}$ & Sí & Profesional \\
\hline
\end{tabular}




\begin{tabular}{|c|c|c|c|c|c|}
\hline Sexo & Edad & $\begin{array}{l}\text { Tiempo en la } \\
\text { organización }\end{array}$ & $\begin{array}{l}\text { Tipo de } \\
\text { organización }\end{array}$ & $\begin{array}{l}\text { Experiencia } \\
\text { previa }\end{array}$ & Ocupación \\
\hline Hombre & 22 & 1 año & $\begin{array}{l}\text { Género y } \\
\text { diversidad } \\
\text { sexual }\end{array}$ & No & Estudiante \\
\hline Mujer & 24 & 2 años y medio & $\begin{array}{l}\text { Derechos } \\
\text { Humanos }\end{array}$ & Sí & Profesional \\
\hline Mujer & 23 & 10 meses & $\begin{array}{l}\text { Educación } \\
\text { Popular }\end{array}$ & Sí & Estudiante \\
\hline Mujer & 26 & 7 meses & $\begin{array}{l}\text { Defensa de los } \\
\text { animales }\end{array}$ & Sí & Profesional \\
\hline
\end{tabular}

El grupo de participantes se conformó bajo el criterio de saturación, debido que éste permitió organizar la información recolectada en temáticas consensuadas. Así, se seleccionó solo a jóvenes universitarios, pues la universidad facilita experiencias de reflexión sobre asuntos públicos, canalizadas en representación estudiantil o voluntariados (Krauskopf, 2000).

Como indican los códigos de ética en investigación (APA, 2017; Goodwin, 2010), a estas personas se les solicitó su consentimiento para participar voluntariamente en el estudio, de manera oral y escrita, y se les comunicó el objetivo, duración y grabación de la entrevista (ver Apéndice A). La confidencialidad de la información y anonimato se aseguró bajo seudónimos. Cada entrevista fue realizada en lugares cómodos, sin lugar a distracciones y acorde a la disponibilidad de los entrevistados. Según Jave y colegas (2015), algunas experiencias participativas implican afrontar situaciones de violencia y estigma; por ello, se diseńó un protocolo de contención: escuchar su vivencia, preguntar por su estado de ánimo, realizar señalamientos positivos de su experiencia, consultar si desea seguir con la entrevista y derivar a un especialista si fuera necesario. Solo se registró un caso de conmoción personal durante el trabajo de campo. Luego de aplicar el protocolo de contención, el participante se sintió aliviado y decidió continuar con la entrevista. 


\section{Técnica de recojo de información}

Se elaboró una ficha de datos sociodemográficos y una guía de entrevista semi-estructurada (ver Apéndice C) para recolectar información acerca de la PP, que cuenta con cuatro ejes de indagación: 1) Repertorios de PP, 2) Motivos y razones de la PP, 3) Significados positivos y negativos de la experiencia de PP, y 4) Percepción sobre la PP de sus pares. La construcción de la guía se basó en la revisión bibliográfica del análisis socio-cognitivo de la PP, y en función a las sugerencias de especialistas en Psicología Política de Perú y Argentina.

\section{Procedimiento}

Primero, se realizó un piloto con dos personas miembros de partidos universitarios, que no formaban parte del grupo final de participantes. Esta aplicación preliminar sirve para evaluar la compresión, organización y fluidez de las preguntas, y para verificar si la información recogida respondía al objetivo del estudio. Una vez concluida la versión final de la guía, se realizó una convocatoria a través de las redes sociales a los jóvenes de distintas organizaciones estudiantiles universitarias vinculadas a la visibilización de problemáticas sociales. Acto seguido, se procedió a coordinar las reuniones con los participantes interesados mediante correos, llamadas telefónicas o de manera presencial. Cada entrevista duró entre 50 y 80 minutos.

\section{Análisis de información}

Las narrativas fueron analizadas con el programa Atlas.Ti. 7.0. El procesamiento de la información se basa en el análisis temático, tanto de carácter deductivo como inductivo, según las sugerencias de Braun y Clarke (2006). Se inició con la familiarización de la data recopilada, al mismo tiempo, se documentaban ideas asociadas a la teoría y potenciales códigos. Luego, siguió el proceso de codificación abierta para identificar categorías emergentes vinculadas a repertorios de PP y su significado para los participantes, con el fin de establecer una lógica inductiva sobre el fenómeno de interés. Posteriormente, se procedió 
con la agrupación de categorías en temáticas iniciales, y en diferentes niveles de abstracción. En concordancia con la integridad del análisis temático, cada temática implicaba una revisión crítica de las transcripciones y su relación con los códigos establecidos de ida y vuelta (Nowell, Norris, White \& Moules, 2017). En base a los ejes de indagación, se fue construyendo las relaciones entre las categorías respaldadas por las teorías psicosociales y las temáticas emergentes, en función a sus similitudes y diferencias, hasta formar un eje central de mayor abstracción y densidad explicativa (Braun \& Clarke, 2006; Clarke \& Braun, 2013).

\section{Resultados y discusión}

Se presentan y discuten los principales resultados del primer estudio, a través de tres ejes: (1) las creencias y repertorios de la PP de los jóvenes; (2) los motivos y atribuciones de la PP de los jóvenes; y (3) los costos y limitantes de la PP.

Cabe resaltar que el análisis de los resultados debe considerarse a la luz de las características de los participantes. Si bien se aprecia una distribución equitativa según sexo, edad, y tipo de organización, la mitad de ellos son universitarios, casi todos dependen económicamente de sus familias, y representan a menos del 8\% de limeńos de 18 a 29 años que han hecho algún tipo de PP (Lima Como Vamos, 2018).

\section{Creencias y repertorios sobre la participación política}

El discurso recogido sobre las características que definen la PP para los jóvenes gira en torno a tres áreas temáticas no excluyentes: La búsqueda de generar un cambio en la sociedad, acciones orientadas hacia las autoridades, y acciones orientadas a la expresión (ver Figura 1). 


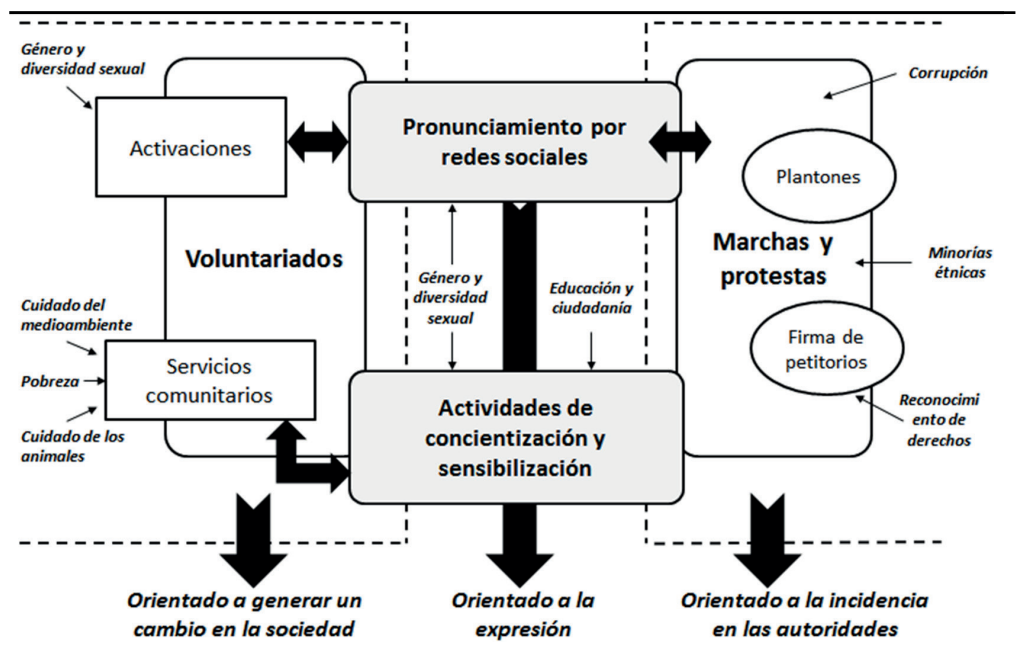

Figura 1

En primer lugar, como aspecto nuclear de la PP, a los participantes les resulta imposible concebir esta sin incorporar la intención propositiva de transformar la realidad social, en otras palabras, de generar un aporte que beneficie a otros, ya sea de manera individual o colectiva. Estas acciones pueden darse a nivel macro, a través de actividades organizadas e institucionalizadas a mediano o largo plazo, o bien, a nivel micro con acciones personales coherentes con sus valores culturales de la sociedad. Además, espontáneamente el dominio de lo político de la PP se aleja de los canales convencionales. Este resultado coincide con varios autores que conceptualizan a la PP en función a su intencionalidad (Conge, 1998; Delfino \& Zubieta, 2010; Vázquez et al., 2006; van Deth, 2014). Por tanto, la creencia sobre PP puede arraigarse en una dimensión de incidencia y en referencia hacia un objeto particular del mundo político.

"Yo creo que una parte si es participación politica y una parte no lo es. Una parte si, porque siento que realizas un cambio y todo cambio que puedas realizar a nivel pequeño se puede convertir a nivel macro, entonces, si hay una participación politica" (Mujer, 21) 
El alcance de la incidencia de la PP juvenil puede organizarse en tres modalidades como se aprecia en la Figura 1: Primero, la PP desde la perspectiva de los entrevistados se concibe como una acción orientada hacia un actor responsable de la situación actual del país, sobre todo, dirigido a autoridades e instituciones políticas. Dentro de esta modalidad de PP, las marchas y protestas son los repertorios percibidos como los más recurrentes y visibles. Si bien estas formas de PP se articulan colectivamente, la decisión de participar es evaluada individualmente, pues se valora el colectivo, en la medida en que les permite expresar su voz, y genera la percepción de que su participación será útil para generar algún cambio esperado.

"Lo que más observo es que los jóvenes van a marchas, esta época ha sido full marchas, desde las marchas contra el indulto de Fujimori por PPK [Pedro Pablo Kuczynski] en los días festivos, más que todo eso. He visto bastante gente de la universidad participar en las [marchas]" (Mujer, 21)

Este punto de vista de los participantes guarda coherencia con los estudios que señalan que, a pesar de la baja PP de jóvenes de 18 a 25 años, la mayoría de ellos acude a marchas y protestas (Lima Como Vamos 2018). Este repertorio parte de una lógica más contenciosa y contestataria para involucrarse con la política, a raíz del descrédito generalizado hacia las instituciones políticas tradicionales, jerárquicas, verticales y burocráticas que no logran resultados favorables para la calidad de vida de los jóvenes (Aguilera, 2010; Arias-Cardona \& Alvarado, 2015; Berreota \& Sandoval, 2014; Nuñera, 2015). Estos resultados guardan relación con las formas de participación de jóvenes de zonas andinas o rurales (Cubides et al., 2015). A partir de un grado de conciencia acerca de los problemas sociales, sus temas de preocupación (Figura 1) giran en torno a la corrupción, la desigualdad de género, los derechos de minorías, y la memoria de la violencia política en el Perú. Estas temáticas de PP juvenil guarda relación con lo encontrado también en estudios latinoamericanos (Cubides et al., 2015). 
A parte de las marchas, otros repertorios de modalidad contestataria consisten en la firma de petitorios, cuyo objetivo es apoyar alguna causa social o demanda ciudadana. Se realizan tanto en el espacio público físico, por ejemplo, en parques, calles y plazas con mayor concurrencia de gente, como en plataformas virtuales, a través de redes sociales digitales y páginas web (Domínguez, López \& Ortiz-Henderson, 2017; Gil de Zúñiga et al., 2014). Cabe mencionar que los petitorios de firmas por Internet suelen ser más comunes y de fácil acceso para los jóvenes entrevistados.

"Por ejemplo, desde Amnistía, ya no estoy tan metida de manera presencial ni asistiendo a muchas de sus reuniones, más bien soy una ciberactivista, que es justo desde las mismas redes sociales puedo apoyar y realizar pedidos por causas con las que simpatizo, [desde Amnistía] y también con la recolección de firmas" (Mujer, 25)

El segundo modo en que se puede articular la PP es a través de acciones de incidencia orientadas hacia la sociedad en general, cuyo espectro político se vincula al bien común (Sorribas \& Brussino, 2017; van Deth, 2014). En dicha modalidad, se encuentran los voluntariados, articulados en servicios comunitarios y activaciones. Ambos repertorios están organizados colectivamente en el espacio público cuya incidencia se centra en generar un beneficio directo a otros. De hecho, se relacionan con las agendas de colectivos activistas que buscan difundir su postura y generar conciencia sobre problemáticas sociales actuales. Así este repertorios de PP permiten dejar en claro la postura de los jóvenes respecto a qué modelo de sociedad peruana aspiran como ciudadanos.

"Dentro de eso, nos reunimos semanalmente acerca de los acuerdos y planificamos nuestras intervenciones, realizamos intervenciones pedagógicas-artísticas, por ejemplo, en nuestro último proyecto, hicimos una alianza con la junta comunal para ver cuáles son los temas que puedan afectar al parque, y detectamos que el problema era medioambiental, entonces, diseñábamos sesiones para que los niños puedan ver ese tema" (Mujer, 23 ańos) 
Lo anterior dialoga con los estudios que problematizan la amplitud del dominio político de la PP (van Deth, 2016), el cual no estaría restringido al vínculo con las instituciones políticas (militancia partidaria y campañas electorales), sino que se extendería al dominio cívico y ciudadano, donde se opta por repertorios participativos que atiendan agendas que critican las visiones culturales predominantes en la sociedad (Cubides et al., 2015; Delfino \& Zubieta, 2010; Sorribas \& Brussino, 2013), sobre todo alineado a sus temas de interés como pobreza, género y diversidad sexual, cuidado del medioambiente y de los animales.

Si bien estas dos modalidades de incidencia son centrales para la PP, porque se orienta a resolver una problemática que afecta a la sociedad, o a un sector de ella, no es lo único que la caracteriza. Para los jóvenes, una tercera forma de PP estaría basada en una dimensión de expresión, así la construcción de una postura u opinión política frente a los temas públicos y controversiales es considerada como acto político válido. Este resultado se alinea con los hallazgos sobre la importancia que los jóvenes otorgan a manifestarse y expresar su voz, principalmente, a través de las plataformas virtuales (Gil de Zúniga \& Valenzuela, 2012; Gil de Zúñiga et al., 2014).

"De ahi, desde la participación individual, los posicionamientos se evidencian desde las redes sociales. Hacer un post sobre yo creo que esto está mal o está bien', o el compartir algo, creo que eso ya supone cierta participación, y el ignorarlo ya no implica una participación activa de la persona" (Hombre 22 años)

En ese sentido, las acciones como los pronunciamientos y el compartir contenidos de opinión pública en las redes sociales indican que estas plataformas dejaron de ser un medio de comunicación interpersonal, para convertirse en el espacio "público" predilecto de expresión, confrontación y potenciación de la PP, como reportan los estudios en Chile y México (Domínguez et al., 2017). En específico, más de la mitad de entrevistados emite su postura frente a temas polémicos en nuestra sociedad, comparte noticias de contenido político y social, 
o publica fotografías que expresan el descrédito de las autoridades políticas. Por tanto, las plataformas digitales adquieren un nuevo significado en el marco de la acción política, como señala Gil de Zuñiga y Valenzuela (2012), en ellas los jóvenes tendrían oportunidades de ampliar sus redes de intercambio de información, de contacto con personas afines a sus intereses, y de demostrar coherencia entre su opinión personal y sus convicciones morales.

"También se ha visto [la participación] desde redes sociales que sirven como una herramienta para la movilización y participación, y hay una mayor crítica hacia las problemáticas que vivimos como sociedad, ya sea desde la violencia hacia la mujer, el tema de la corrupción y hay un mayor interés por estos problemas sociales" (Hombre, 22 años)

$\mathrm{Al}$ respecto, la expresión de sus posturas ante asuntos públicos no se agota en las redes sociales, sino que necesitaría canalizarse de manera organizada en espacios físicos, como las actividades de sensibilización. Como mencionan los entrevistados, la mayoría realiza talleres o actividades informativas: publicación de posters, pancartas o afiches en la vía pública, o lugares donde se congregue la mayor cantidad de gente, cuyo propósito es concientizar sobre una problemática social a través de información o mensajes disonantes.

"Otra actividad fue tomar el espacio público con carteles sobre violencia, apelando a títulos como "Tú puedes ser la siguiente, Mira lo que está pasando con las mujeres" para fomentar reflexión y recién el siguiente ciclo hacer una actividad respaldad por la universidad sobre temas de diversidad sexual" (Hombre, 22 años)

En menor mención, otra agrupación de actividades de sensibilización son los talleres interactivos. En ellos, se despliegan el uso de herramientas artísticas en plazas y parques; por ejemplo, la implementación de micro-teatros, el uso de instrumentos musicales o afiches que aluden a las consecuencias negativas del comportamiento humano sobre el medioambiente y la sociedad. Usualmente, están dirigidos a niños y jóvenes, con el propósito de generar diálogo y reflexionar sobre su rol como ciudadanos. 
"La otra vez con el grupo armamos pequeñas presentaciones de teatro dirigido a niños, hay de todo, guitarras, charangos, cánticos, para pensar en lo que hacemos cuidando nuestro espacio [público], terminamos sentados en el parque y hacemos preguntas sobre qué sociedad queremos; asi a través de la educación popular tratamos de hacer un pequeño cambio" (Mujer, 23 ańos)

A partir de lo anterior, se puede inferir que las modalidades de PP juvenil se expanden a nuevas formas creativas de vincularse con el dominio político, en este caso, a partir de la interacción directa con una comunidad y empleando recursos artísticos que buscan incidir en el espacio público, buscar un cambio en su localidad, o problematizar situaciones normalizadas, de manera organizada. Así, este repertorio puede entenderse como una expresión de la búsqueda de un estilo de participación política menos jerárquica y más horizontal (Nuñera, 2015).

\section{Motivos y atribuciones de la participación politica}

Las narrativas sobre por qué los jóvenes toman la decisión de participar políticamente se vinculan, sobre todo a los repertorios más frecuentes y visibles de la PP, como es el caso de las marchas, el voluntariado y pronunciamientos.

De ese modo, se ha organizado la data discursiva en función a cuatro ejes que representan los motivos de la PP: el interés, conciencia e indignación por la situación de la sociedad, el rol de los pares, la exploración de la identidad, y el beneficio personal y/o profesional (ver Figura 2).

Para los pronunciamientos y marchas, el núcleo motivacional de los participantes alude a un aspecto afectivo de la participación, entendida como la preocupación por los temas de agenda pública que potencialmente pueden afectar la calidad de vida de ellos mismos, sus pares y de la sociedad en general. Este interés estaría estimulado por el contexto universitario, pues ofrece oportunidades de deliberación y reflexión como ciudadanos a través de la política universitaria y proyectos de responsabilidad social (Gasca-Pliego \& Olvera-García, 2011). 


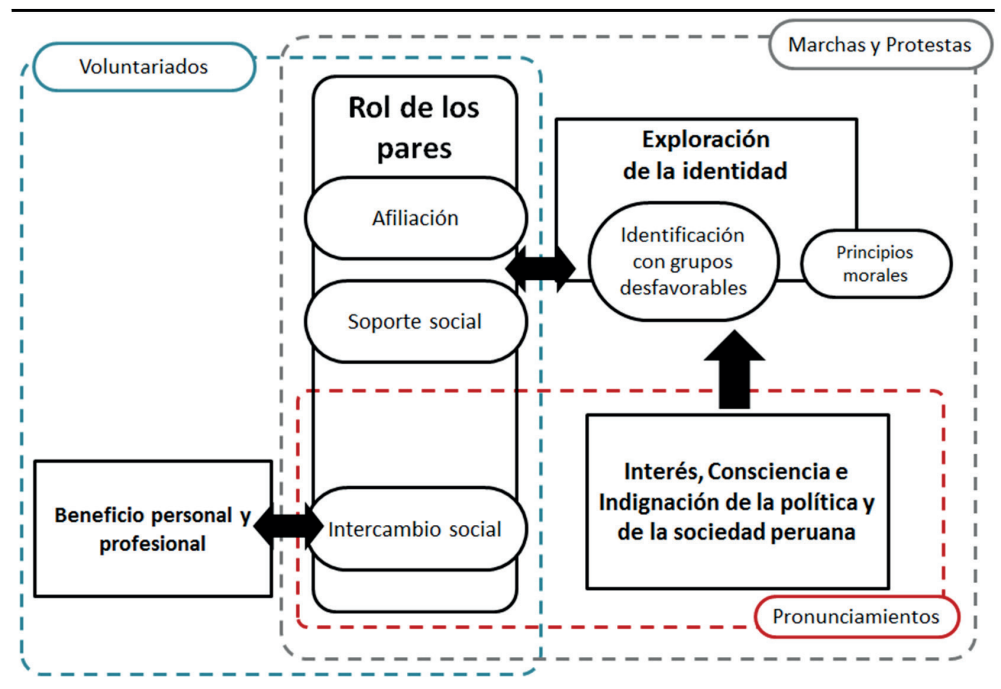

Figura 2

Al mismo tiempo, como seńala varios autores (Ames, 2013; AriasCardona \& Alvarado, 2015; Botero et al., 2008), se corrobora que los jóvenes no serían necesariamente personas apáticas e insensibles del acontecer social y político del país, sino que, en base a los medios de comunicación y las redes digitales, van elaborando su propia percepción del funcionamiento y dinámica de la sociedad. Este resultado coincide con estudios sobre sofisticación política como antecedente de la PP (Brussino et al. 2011; Grönlund \& Milner, 2006), que encuentran que los jóvenes con mayor nivel de PP muestran mayor atención, conocimiento e interés en la política

"Creo que hay una mayor comprensión y crítica hacia las causas sociales que vivimos en la actualidad, ya sea el tema de violencia basada en género, el tema de la corrupción actual; hay un mayor interés por estas problemáticas sociales" (Mujer 25 años) 
En ese sentido, el acontecer social y político es un objeto actitudinal que tendría efecto sobre el involucramiento en marchas y pronunciamientos. Los participantes muestran una actitud negativa generalizada hacia el sistema político, pues es visto como carente de legitimidad, respeto y honestidad. En sintonía con dicha actitud, ellos experimentan un estado emocional de indignación por los sucesos públicos que los afectan como miembros de la sociedad, siendo la corrupción el ejemplo más señalado. Estos resultados coinciden la percepción de un sistema corrupto y proclive de avalar la trasgresión a la norma sería un movilizador de acciones contenciosas y un canal para expresar su descontento (Janos et al., 2018; Jorge, 2016), como se aprecia en la siguiente cita.

"Creo que también hay una cuestión de coyuntura, creo que ahorita hay un contexto intenso de corrupción y violencia, de cosas que están mal en la sociedad, y se ha vuelto tan evidente y creo que es imposible que un sector de la sociedad no se movilice frente a eso" (Hombre, 22 años)

Dicho estado emocional, sería también resultado del conocimiento e interés por la situación de grupos socialmente vulnerables en la sociedad peruana. Este correlato prosocial deriva de tomar conciencia sobre la opresión ejercida hacia grupos de bajo estatus social, y en sentir empatía hacia los mismos, quienes son evaluados como víctimas de injusticias, pues, como señalan varios estudios, la ira lleva a las personas a participar en repertorios de PP no convencional (Sabucedo \& Vilas, 2014; Sandoval \& Silva, 2016; van Zomeren et al., 2008; Vázquez et al., 2006). Así, guarda sentido que la participación en marchas y pronunciamientos sean una forma de expresión y preocupación por los grupos históricamente minusvalorados en la sociedad peruana (mujeres, comunidad LGTBI, o minorías étnicas).

"En activismo, por lo que veo, las luchas parten de luchas propias, por ejemplo, una mujer puede decir: 'yo como mujer me doy cuenta de que existe el patriarcado y me violentan, empiezo a investigar más, y tomo consciencia, y empiezo mi lucha'. En ese sentido, en Manada Feminista, casi todas son mujeres y conscientes de esa opresión que viven" (Hombre, 24 años) 
En el caso particular de las marchas y voluntariados, la PP juvenil no se restringe a motivos prosociales ni emocionales, sino que resulta aún más importante comprender los motivos interpersonales que facilitan la implicación de los jóvenes en este tipo acciones.

Los resultados revelan que los pares serían un elemento clave para canalizar el acercamiento de los jóvenes hacia repertorios de PP. Cada una de las organizaciones civiles es considerado como un grupo potencialmente atractivo para satisfacer la necesidad de afiliación y pertenencia. En coherencia con la teoría de necesidades psicológicas básicas (Ryan \& Deci, 2017), el hecho de participar como miembros de colectivos permite satisfacer la necesidad de relación, porque ofrecería una oportunidad para conocer y sentirse conectado a otros pares con intereses, inquietudes y preocupaciones similares. Así, como mencionan los estudios sobre identidad social y PP (Sabucedo et al., 2010; van Stekelenburg et al., 2011), el grupo resulta una fuente motivacional donde convergen las simpatías en común, cuestionamientos y propósitos compartidos, y que originan las acciones de PP; de ese modo, para los jóvenes resultaría atractivo ser parte de un colectivo homogéneo, con el cual puedan identificarse y compartir temas en común, como se aprecia en el siguiente relato.

"Sentía que habia diversas causas que me movilizaban y que no encontraba un lugar o un grupo en cual poder encausar. Sentía a veces que la misma dinámica de la universidad podía atomizar muchas cosas y esa necesidad de un grupo que también pensara las mismas cosas que uno y [poder] generar un cambio" (Mujer, 25 ańos).

Como indica la Figura 2, si bien el grupo de pares por sí mismo es una motivación para involucrarse en acciones de marchas y voluntariados, para una pequeńa parte de los entrevistados, participar es también una oportunidad de brindar soporte y apoyo social a personas de su entorno cercano. El respaldo a familiares o amigos afectados, física o simbólicamente por problemáticas sociales, sería una oportunidad para validar y afianzar sus vínculos interpersonales. Por lo tanto, los jóvenes se ven más dispuestos a participar si el repertorio de PP 
facilita un mayor involucramiento en la construcción de vínculos interpersonales y en demostrar mayor sensibilidad ante eventos públicos que atentan contra el bienestar e integridad de sus amigos o familiares, como se expresa en la siguiente cita.

"El poder hacer algo por ese grupo o por esa persona, para mi es como... wau, no estás sola, como una actividad que hicimos por Máxima Acuña, no estás sola, esa persona no está sola. Hay una comunidad muy grande de personas que quieren apoyarte y están dispuestas a hacerlo, creo que ese sentido de humanidad que muchas veces perdemos [...], pero creo que me genera mucho a mi el que puedo también servir, [...] y poder estar en disposición para servir a alguien creo que es algo valioso que la persona puede tener y si puedes ayudar a que alguien más también pueda ser así, genial" (Mujer, 24 años)

Las motivaciones interpersonales de la PP juvenil no solo residen en el rol de los pares, sino en las oportunidades para la exploración y formación de identidad, que contribuye al desarrollo interpersonal (ver Figura 2). Para la mayoría de los participantes que acuden a protestas, un gran incentivo de participación sería en que esta permite un proceso de conocimiento de sí mismo, con el fin de validar y confrontar su propia identidad dentro de un contexto percibido como conflictivo, y que cuestionan sus valores personales.

"a veces cuando algo te toca de manera personal quieres de todas maneras que haya un cambio en la sociedad para que no se den más estas situaciones de violencia (...) porque, por ejemplo, yo me involucre en el voluntariado, y de hecho me involucre bastante en temas de género porque en algún momento llegué a cuestionar mi sexualidad, y es por eso averigüé más sobre estos temas de las luchas LGTBI, de la orientación sexual, de los crimenes de odio, y fue algo que me toco de manera personal" (Hombre, 22 años)

La anterior cita ejemplifica que los grupos víctimas de violencia y perjuicio podrían servir como un referente para el cuestionamiento sobre sí mismos en la medida en que parte de la subjetividad de los jóvenes se interpela, y se identifican con ellos. Este proceso de identi- 
ficación, según varios autores, canaliza a un estado motivacional que potencialmente los incita a participar a favor del grupo afectado (Sabucedo, 1988; Sandoval, 2001; van Stekelenburg \& Klandermans, 2010; van Zomeren et al., 2008). Para los entrevistados, las marchas por el reconocimiento de derechos y justicia serían consideradas como una forma de "lucha personal".

En adición, el motivo de formar y confrontar su identidad, para un par de participantes, no se limita necesariamente al canal de identificación con otros, sino que también incluye la construcción subjetiva de las propias convicciones morales. En ese sentido, participar sería una oportunidad para actuar acorde y coherentemente a los valores personales que aspiran tener, en este caso, la igualdad e inclusión. Según varios estudios psicológicos (Porter, 2013; Sabucedo et al., 2018; van Stekelenburg, 2013; Youniss, 2009), la PP sería una vía de reafirmación de los intereses morales, sirviendo de anclaje para la maduración de la identidad, sobre todo, a través de las formas expresivas de participación, como podrían ser las marchas. Así, el colectivo del cual forman parte los participantes es valorado, en la medida en que les permite satisfacer ese sentido de coherencia moral entre lo que piensan, valoran y hacen; como se aprecia en la siguiente cita.

"Digamos que son bien ligados a esta lucha feminista, reconocimiento por minorias, inclusión; había bastantes [ideas] relacionados a la no discriminación y a tratar de ser más intercultural. Me llamó bastante la atención, y a pesar de que sentía que recién estaba formando esos valores y que no todos los seguía al pie de la letra, sentía que al formar parte de ese grupo iba a terminar de construirme como yo quería ser. Entonces, participé y tratamos de que todas las actividades tengan relación con los lineamientos que tenemos" (Mujer 21 años).

Hasta el momento, las distintas motivaciones de la PP, sobre todo en protestas y actividades de sensibilización, se han centrado en la preocupación por otros y en su contribución para el desarrollo de la identidad personal. No obstante, también emerge un aspecto motiva- 
cional más instrumental, bajo una lógica de costos y beneficios acorde a sus intereses personales.

"También a veces porque para el tema de conseguir un trabajo a veces piden como requisito que uno haya estado en voluntariados. De hecho, recuerdo que a una feria de voluntariados a la que asisti, hace ya bastante tiempo, me acuerdo que habia voluntariados del estado y ellos explícitamente te decian, sería bueno que te metas a esto voluntariado porque al momento de dar becas para maestría del estado, te damos más puntos si estas en un voluntariado nacional" (Hombre, 22 años)

En relación con el anterior discurso, la mitad de los participantes considera que el beneficio personal entra en la dinámica de motivadores que afecta la decisión de formar parte de voluntariados (ver Figura 2). Esta motivación orienta a los jóvenes a buscar actividades de participación que sean fuente de desarrollo de competencias personales y profesionales, pues estas serían útiles para realizar las tareas correspondientes a su siguiente etapa de desarrollo: la finalización de estudios, el primer empleo, u otras tareas de mayor responsabilidad e independencia; las cuales son metas deseables y valoradas socialmente entre las personas de 20 a 29 años de zonas urbanas (Uriarte, 2005).

\section{Limitantes y costos de la participación política}

A parte de las atribuciones y motivos presentados, la decisión de participar puede depender del balance entre los costos, los beneficios y los recursos requeridos para desenvolverse como agentes políticos. En ese sentido, las respuestas de los participantes se han organizado del siguiente modo: la carga académica y responsabilidades, el desinterés por la política y las problemáticas sociales, la desesperanza de lograr un cambio, el perjuicio a la integridad física y psicológica, y la oposición del entorno familiar (Ver Figura 3). 


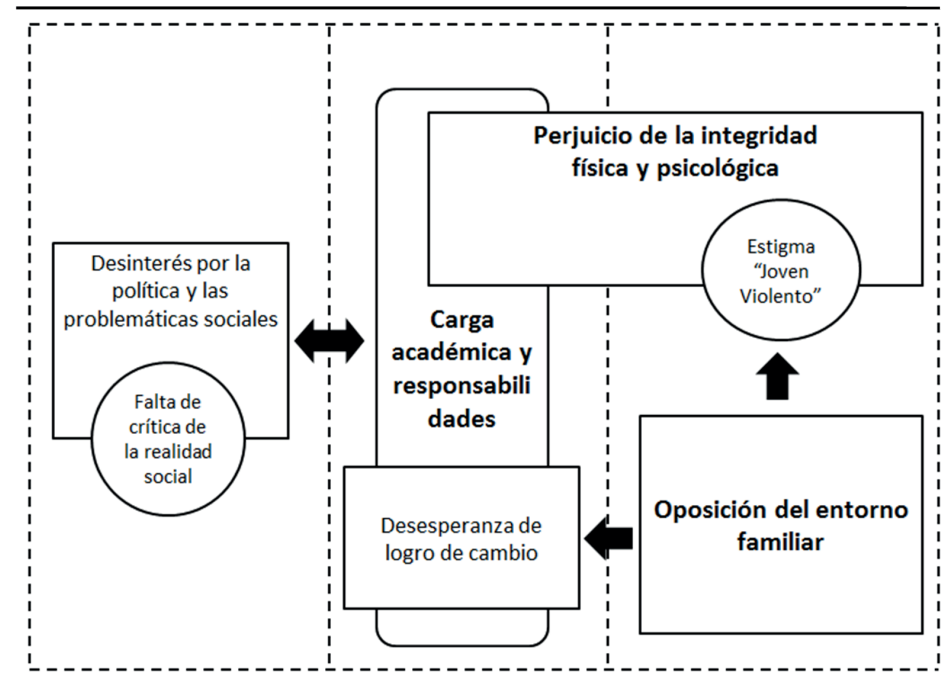

Figura 3

Desde la lógica de costo-beneficio de la participación y movilización (Klandermans, 1984; van Stekelenburg \& Klandersman, 2010), se puede inferir que la PP compite con otras actividades de igual o mayor importancia para los jóvenes. Como la mayoría son universitarios, lidian con deberes estudiantiles, tales como proyectos, lecturas, o trabajos grupales; por tanto, el tiempo para involucrarse en las acciones de PP depende de la carga académica, y de su capacidad para organizar sus horarios y balancear sus obligaciones. A su vez, la responsabilidad y esfuerzo de organizar las marchas serían otro costo personal, pues dichos repertorios, a veces, implican procesos burocráticos con autoridades, así como desarrollar estrategias de comunicación, desplazamiento y cuidado de los asistentes. Esta organización es vista como tediosa y prefieren evitarla; incluso, se siente inseguros cuando asumen roles de coordinación y deben ser responsables de las acciones de otros. Junto a la carga académica, esta responsabilidad sería el principal aspecto disuasivo de PP, como se aprecia en la Figura 3, y en la siguiente cita. 
"Por ejemplo, cuando estaba en [voluntariado], muchos chicos que entraron al voluntariado dejaron de asistir, porque priorizaron sus estudios, y también existen voluntariados que te quitan mucho tiempo... me corrijo, voluntariados demandantes, y bueno, a veces necesitas priorizar, primero, los estudios... y creo que existen jóvenes que no se mueven si no les das un sol" (Mujer, 23 años)

Por un lado, se mencionó que la indignación por injusticias sería un motivador de PP juvenil; sin embargo, si dicha emoción no encuentra una vía de expresión conductual, podría acabar siendo una limitación de la PP en marchas y pronunciamiento. En efecto, si dichos repertorios no logran cumplir con los resultados esperados y la indignación de los jóvenes termina en un mero desgaste emocional, se va configurando una percepción de que el sistema político está estancado, y que la sociedad no tiene futuro. En congruencia, los jóvenes terminan por evaluar cualquier intento de participación como un costo inútil a corto plazo, y empiezan por creer que no tienen la capacidad necesaria para cambiar la situación de su interés, así la participación pierde sentido. Esta percepción de desesperanza se asocia al alto cinismo político y bajo sentido de eficacia, ambos factores son un riesgo para la democracia y potenciales inhibidores de la PP no convencional (Beramendi \& Zubieta, 2013; Jorge, 2016; Mannarini et al., 2009; Pattyn et al., 2012; Sorribas \& Brussino, 2013).

"Siento que [los jóvenes no participan] por el hecho de que sienten que la política es sucia y sienten que no van a cambiar nada, que igual lo van a hacer. Yo [...] conozco gente más adulta que también dice lo mismo, prefieren quedarse en sus casas y no salir a marchas o no proponerse otro tipo de actividades, porque saben que no van a logran nada con [salir a marchar] y que el pais va a seguir igual" (Mujer, 21 años)

Por otro lado, para las mujeres participantes, la violencia percibida en las protestas suele disuadir la decisión de involucrarse en la PP. A partir de la información de los medios de comunicación y de su propia experiencia, las participantes perciben el potencial de ser víctimas de agresión física y psicológica de parte de las fuerzas policiales, de los 
mismos participantes o de personas que están en contra del motivo de participación, lo cual generaría miedo e inseguridad en las participantes, y sería un alto costo que atentaría contra su bienestar. A su vez, esa percepción de violencia alrededor de las marchas se vincula con el estigma del "joven violento": impetuoso, caótico e irruptor del orden social. Ante el escrutinio público, los jóvenes podrían asumir dichas características negativas como parte de su identidad, ergo, sentirse devaluados, y potencialmente rechazados. Este estigma de "violencia" resultaría ser un correlato cognitivo producto de la historia peruana cargada de la violencia política durante los 80 s y 90 s, que se extiende a los universitarios (Jave et al., 2015; Rosas, 2009), limitando de la PP.

"[No volvería a participar] porque es feo y quejoso, la policía estaba tirando bombas lacrimógenas, estaba pegando, arrastrando, metía a los jóvenes al patrullero para arrestarlos, como vandalismo y todas esas cosas... y yo dije: "creo que esa no es la forma de llamar la atención", bueno, tal vez hemos llamado la atención, pero no me gustó la forma, o sea al principio, me pareció que era una marcha de gritar solamente, pero luego se pasó al otro extremo de violencia, y yo creo que con violencia se puede ganar, pero igual trae más violencia, y te generas una mala reputación” (Mujer, 26 años)

Por último, la PP puede traer consigo la oposición del entorno familiar. Para la mayoría, simpatizar con modalidades contenciosas de PP (marchas y protestas) o pronunciarse a favor o en contra de temas polémicos implica confrontar la valoración negativa por parte de personas significativas en sus vidas, como los familiares con postura contraria. Desde la mirada de Klandermans (1984), el valor de los otros, en este caso, la opinión de los seres queridos sería más valorada y supone un mayor costo social, en comparación con el decisión de no involucrarse en marchas, ya que la familia lo vincula a violencia, inseguridad o a pérdida de tiempo. Contingentemente, emerge el temor de perder los vínculos interpersonales cercanos; más aún si la mayoría de los participantes dependen social y económicamente de ellas. 
Por ello, la familia sería otro contexto relacional, en el cual los jóvenes buscan también afianzar sus vínculos y satisfacer su necesidad de relación, y con tal de preservar dichos vínculos, su opinión sería un factor de disuasión y una limitación frente a la PP (van Stekelenburg et al., 2011).

"Simpatizo con el colectivo, pero, si solo cuando denuncio por Facebook o me pongo a favor de la diversidad sexual, tengo problemas... mi familia es conservadora y tradicionalista, y algunos me dicen que no debería hacerlo, se molestan conmigo... recuerdo que en los comentarios se desata opiniones de algunos familiares cercanos que descalifican lo que hacia, te imaginas que pasaría si voy a una marcha por el "Orgullo Gay" y de toda la comunidad LGTBI, sería dificil soportarlo, me sería muy dificil" (Hombre, 22 años)

\section{Estudio 2}

\section{Método}

En el segundo estudio, se tiene como objetivo analizar las relaciones entre las variables socio-cognitivas, actitudinales y emocionales vinculadas a la PP no convencional (PPNC).

\section{Participantes}

La muestra fue 132 jóvenes residentes en Lima, cuyo rango etario oscila entre 18 y 25 años. Cabe mencionar que solo se incluyó jóvenes residentes en Lima Metropolitana, pues existe mayor información de las diversas acciones de PP en esta ciudad (Lima Cómo Vamos, 2018; SENAJU, 2015). Como variables de control, se registró información en función al sexo, nivel educativo, ocupación y nivel socioeconómico de los participantes (Tabla 2). 


\section{Tabla 2}

\section{Características de la muestra}

Variable $\quad$ Total $(N=132)$

Edad (promedio)

$24.5(D E=2.4)$

Sexo

Hombre

$42 \%$

Mujer $58 \%$

Nivel Educativo

Secundaria $14 \%$

Superior Técnico $4 \%$

Superior Universitario Incompleta $60 \%$

Superior Universitario Completa $18 \%$

Posgrado $4 \%$

Ocupación

Estudiantes de Educación Superior $79 \%$ Trabajador Independiente $15 \%$

Trabajador Dependiente $18 \%$

Desempleado $8 \%$

Ni estudia ni trabaja $3 \%$

Nivel Socioeconómico
A
$34 \%$
B
$45 \%$
C
$18 \%$
D
$2 \%$
E
$1 \%$

Asimismo, se registró la adscripción a organizaciones civiles, estudiantiles, religiosas, de género, ecologistas o vecinales, como se aprecia en la Tabla 3. 


\section{Tabla 3}

\% de participantes según tipo de organización adscrita

\begin{tabular}{lc}
\hline \multicolumn{1}{c}{ Organización } & Total $(N=132)$ \\
\hline Religiosa & $13 \%$ \\
De género & $24 \%$ \\
Ecologista o ambientalista & $21 \%$ \\
Vecinales & $43 \%$ \\
Estudiantil & $20 \%$ \\
Civil & $17 \%$ \\
\hline
\end{tabular}

La selección de los participantes se realizó a través de un muestro no probabilístico accidental, específicamente, mediante una convocatoria pública en la plataforma de Facebook.

\section{Medición e instrumentos}

Conocimiento político: Se usó la Escala de Conocimiento Político y Conocimiento Cívico (Brussino, Sorribas \& Medrano, 2008) adaptada al contexto local. Está compuesta de 8 ítems, y evalúa el grado de entendimiento de la dinámica política (e.g. “¿Cuál es la entidad encargada de promulgar leyes?"). Las respuestas varía en 3 opciones, donde 2 = Correcta $; 1$ = Parcialmente Correcta, y $0=$ Incorrecta. El puntaje se obtiene a través de la sumatoria de las respuestas. De hecho, la presente escala muestra una sólida consistencia interna de $\alpha=.77$.

Interés en Política: Mediante la escala de Brussino y colegas (2006), se estima el grado de interés en asuntos políticos (e.g. "Tener conversaciones sobre asuntos políticos y de gobierno"). Esta versión consta de 6 ítems con una escala tipo Likert de 1 a 5, donde 1 = Ningún Interés y 5 = Mucho Interés. Su confiabilidad resulta ser satisfactoria, con un $\alpha=.90$.

Eficacia Política: Se utilizó la Escala de Eficacia Política Interna y Externa (Niemi, Craig \& Matttei, 1991), adaptada en población de 18 a 29 años, mayoritariamente universitarios, por Brussino, Sorribas, Rabbia y Medrano (2006). Se divide en Eficacia Política Interna que 
evalúa la capacidad percibida en asuntos políticos, (e.g. "Podría desempeñarme en un puesto político tan bien como cualquier persona”); y la Eficacia Política Externa, la creencia de que uno es capaz de lograr respuesta de parte del gobierno (e.g. "Tu opinión ejerce alguna influencia sobre las decisiones de las autoridades políticas"). Cada factor consta de 4 ítems con un formato tipo Likert del 1 al 5, siendo 1 = Totalmente en desacuerdo, y 5 = Totalmente de acuerdo. Varios estudios utilizaron esta escala (Sorribas \& Brussino, 2013b; 2017), demostrando su validez. En el estudio, se muestran una robusta confiabilidad de $\alpha=.88$ y $\alpha=.71$, respectivamente.

Confianza Política: Con la escala desarrollada por Hahn (1998), traducida y adaptada lingüísticamente al contexto local, se evalúa las actitudes hacia el gobierno, basados en su desempeño y cumplimiento de las expectativas ciudadanas (e.g. "La mayoría de personas en el gobierno son honestas"). Cuenta con 7 ítems en formato de respuesta tipo Likert de 1 a 5, donde $1=$ Muy en Desacuerdo y $5=$ Muy de Acuerdo. La escala muestra una confiabilidad de $\alpha=.75$.

Cinismo Político: Se evaluó a través de la Escala de Cinismo Político (Janos et al., 2018) validada en distintas ciudades del Perú, y contiene 5 dimensiones: 6 ítems de Desconfianza en el Sistema Político (e.g. "Es irrelevante por quien vote, ya que todos los candidatos son corruptos y esto no va a cambiar"), 3 ítems de Laxitud Moral Política (e.g. "Todos los políticos son malos, así que es mejor elegir a aquellos que "roban pero hacen obras"”), 2 ítems de Corrupción en el Sistema Político (e.g. "La corrupción está muy ligada al sistema político en el país”), 3 ítems de Ineficacia del Sistema Político (e.g. "Siento que el sistema político peruano difícilmente mejorará”), y 2 ítems de Posibilidad de Cambio del Sistema Político (e.g. "Ante un mal sistema político, es responsabilidad de los ciudadanos hacer cosas para cambiarlo"). La escala de respuesta es tipo Likert del 1 al 4 , siendo 1 = Totalmente en desacuerdo, y 4 = Totalmente de acuerdo. La escala global presenta una consistencia interna aceptable de $\alpha=.80$; y cada una de sus dimensiones, una confiabilidad aceptable de $\alpha=.84, \alpha=.69, \alpha=.66$, $\alpha=.41$ y $\alpha=.60$, respectivamente. Dada la baja confiabilidad del factor 
Ineficacia del Sistema Político, no será considerada en el reporte de resultados.

Emociones sobre el contexto sociopolítico: Se aplicó una escala desarrollada para el presente estudio en base a estudios sobre emociones y PP (Jasper, 2011; Páez et al., 2013; Sabucedo \& Vilas, 2014, Sandoval \& Da Silva, 2016; Rico et al., 2017). Dicha escala evalúa el nivel de intensidad emocional personal acerca de la situación sociopolítica de la sociedad, cuenta con 7 ítems (e.g. "Indignación; Esperanza”), donde $1=$ Nada, y 5 = Mucho. Se agrupan en dos factores según su valencia afectiva (Emociones Positivas y Emociones Negativas) con una robusta consistencia interna de $\alpha=.79$, y $\alpha=.80$, respectivamente (ver Apéndice D).

Participación política no convencional: En base al primer estudio y la literatura sobre repertorios de PP (Brussino et al., 2006), se desarrolló una escala con 15 ítems que mide la frecuencia de acciones de PP realizadas en el último ańo. Las respuestas se miden mediante una escala de frecuencia, donde 1 = Nunca, $2=$ Muy pocas veces, 3 = Algunas veces, y $4=$ Muchas veces. Para esta versión se presenta un análisis factorial exploratorio (AFE) para identificar la agrupación de los ítems acorde a la revisión literaria, que se detallará en los resultados.

En relación con el AFE de la escala de Repertorios de Participación Política No Convencional (E-PPNC), los 15 ítems se agrupan en dos factores: PP Expresivo-Cívica y PP Contenciosa, cumpliendo plenamente con los indicadores de bondad del ajuste (ver Tabla 4). El primer factor refiere a las modalidades expresivas, de compromiso cívico y normativo de participación, mientras que el segundo factor alude a los repertorios de instigación, manifestaciones en las calles y reclamo directo en el espacio público. 


\section{Tabla 4}

Análisis factorial exploratorio con rotación oblicua y matrices policóricas de la participación politica no convencional en jóvenes

Ítems

PP Expresivo-

PP

Cívica

Contenciosa

Emitir su opinión, reclamos o quejas a través de

los medios de comunicación masiva (radio, TV,

periódicos, etc.)

Asistir a alguna reunión pública convocada para

discutir problemas sobre el lugar donde vive

0.717

(edificio de residencia, barrio, distrito, etc.)

Participar en actividades o campañas de

sensibilización en las calles, plazas o parques

0.703

Firmar un comunicado o pedidos en la vía pública

(calles, parques, plazas, etc.)

Realizar talleres en el espacio público sobre temas relacionados con el ejercicio de la ciudadanía

0.535

Emitir pronunciamientos por redes sociales sobre

temas públicos o de actualidad política

Compartir comentarios o noticias sobre temas

políticos por redes sociales (Facebook, Twitter, etc.)

Firmar un comunicado o pedidos por Internet

Organizar la firma de peticiones sobre temas públicos (en la calle o en internet)

0.364

Participar de algún voluntariado 0.355

Bloquear pistas o carreteras

0.975

Organizar marchas o plantones pacíficos

0.868

Asistir a marchas y/o protestas

0.697

Participar en plantones pacíficos en la calle, plazas o parques

Participar en la toma de oficinas o locales públicos

$-$




\begin{tabular}{|c|c|c|}
\hline Ítems & $\begin{array}{l}\text { PP Expresivo- } \\
\text { Cívica }\end{array}$ & $\begin{array}{c}\text { PP } \\
\text { Contenciosa }\end{array}$ \\
\hline$\alpha$ de Cronbach Global & \multicolumn{2}{|l|}{.883} \\
\hline $\mathrm{KMO}$ & \multicolumn{2}{|l|}{.821} \\
\hline Prueba de esfericidad de Bartlett & \multicolumn{2}{|l|}{$908.0^{* * *}$} \\
\hline Varianza explicada & \multicolumn{2}{|l|}{$58.27 \%$} \\
\hline CFI & \multicolumn{2}{|c|}{.982 (>.90 = Buen Ajuste $)$} \\
\hline NNFI & \multicolumn{2}{|c|}{$.975(>.90=$ Buen Ajuste $)$} \\
\hline RSMEA & \multicolumn{2}{|c|}{$.072(<.08=$ Buen Ajuste $)$} \\
\hline${ }^{* * *} \mathrm{p}<.001$ & & \\
\hline
\end{tabular}

\section{Procedimiento}

Se inició con la aplicación del piloto a 17 universitarios para corroborar la comprensión de los ítems, e identificar posibles mejoras al protocolo; luego, se siguió con el análisis estadístico preliminar. En base a los contactos establecidos en el primer estudio, se realizaron coordinaciones para el levantamiento de información, con el fin de convocar, a través de correos y Facebook, a jóvenes interesados en participar del estudio y que cumplan con el perfil de 18 a 25 años y residentes en Lima. Cada participante accedió a la encuesta online mediante un enlace de Survey Monkey. En la primera sección del protocolo online, se presentó el consentimiento informado (ver Apéndice B) donde se explica el objetivo y los alcances del estudio, haciendo énfasis en la participación voluntaria y confidencialidad de la información recogida, así como dando los datos de contacto del investigador principal para absolver dudas.

\section{Análisis de Datos}

La data se analizó con el SPSS 22.0. Con el fin de evaluar la normalidad de los datos, se calculó los índices de curtosis y asimetría, mostrando valores adecuados para análisis multivariado $( \pm 3)$. En caso 
del AFE de la E-PPNC, se utilizó Factor (Lorenzo-Seva \& Ferrando, 2006; 2019) que permite realizar el análisis bajo matrices policóricas y métodos de extracción de factores más robustos. Su análisis está sujeto a los siguientes indicadores de bondad de ajuste (Kline, 2011): el índice de ajuste comparativo (CFI) y el índice normalizado de Bentler-Bonett (NFI) y la raíz del error cuadrático medio de aproximación (RMSEA) de Steiger-Lind. Se consideraron como valores mínimos de un adecuado ajuste: CFI y NFI > 0,9, y RMSEA < 0,08. Además, se realizaron correlaciones y análisis de senderos (path analysis) con los puntajes globales de las variables psicológicas sobre PP. La evaluación de los modelos de senderos se basa en los mismos criterios.

\section{Resultados}

\section{Descriptivos de las variables}

A nivel descriptivo, se presentan puntuaciones medias y altas en las distintas medidas socio-cognitivas y actitudinales de Conocimiento Político, Interés en Política, Eficacia Política Interna y Cinismo Político (ver la Tabla 5). No obstante, se aprecia bajos niveles de Eficacia Política Externa, y Confianza Política. Sobre las medidas emocionales, se registra un puntaje alto de Emociones negativas, y un nivel medio bajo de Emociones Positivas, con excepción de la Esperanza. Respecto a la frecuencia de la PP no convencional, la PP Expresivo-Cívica suele ser medianamente frecuente, mientras que la frecuencia de PP Contenciosa es baja (ver Tabla 5). 


\section{Tabla 5}

Descriptivos generales de la variables sociocognitivas, actitudinales, emocionales y de participación politica

\begin{tabular}{|c|c|c|c|c|c|c|c|}
\hline \multirow{2}{*}{ Variable } & \multicolumn{3}{|c|}{ Descriptivos } & \multicolumn{4}{|c|}{ Normalidad } \\
\hline & $M$ & $M e$ & $D E$ & $z$ & $p$ & $g 1$ & $g 2$ \\
\hline Interés en Política & 3.63 & 3.67 & 0.91 & .083 & .027 & -0.42 & -0.30 \\
\hline Conocimiento Político & 8.07 & 8.00 & 3.96 & .098 & .003 & -0.53 & -0.23 \\
\hline Eficacia Política Interna & 3.34 & 3.50 & 1.09 & .081 & .034 & -0.35 & -0.56 \\
\hline Eficacia Política Externa & 2.68 & 2.75 & 0.74 & .083 & .028 & 0.25 & 0.31 \\
\hline Confianza Política & 2.12 & 2.14 & 0.61 & .088 & .013 & 0.31 & 0.72 \\
\hline Cinismo político & 2.34 & 2.31 & 0.35 & .086 & .018 & 0.03 & 0.56 \\
\hline $\begin{array}{l}\text { Desconfianza en el sistema } \\
\text { político }\end{array}$ & 2.18 & 2.16 & 0.57 & .130 & .000 & 0.29 & 0.65 \\
\hline Laxitud moral política & 1.68 & 1.67 & 0.58 & .171 & .000 & 0.88 & 0.94 \\
\hline $\begin{array}{l}\text { Corrupción en el sistema } \\
\text { político }\end{array}$ & 3.45 & 3.50 & 0.48 & .217 & .000 & -0.56 & 0.40 \\
\hline Posibilidad de cambio & 1.64 & 1.50 & 0.54 & .198 & .000 & 0.33 & -0.79 \\
\hline $\begin{array}{l}\text { Emociones positivas de la } \\
\text { situación social y política }\end{array}$ & 2.71 & 2.67 & 0.96 & .094 & .006 & 0.24 & -0.29 \\
\hline Esperanza & 3.16 & 3.00 & 1.14 & .164 & .000 & -0.13 & -0.69 \\
\hline Optimismo & 2.89 & 3.00 & 1.05 & .210 & .000 & 0.09 & -0.32 \\
\hline Orgullo & 2.08 & 2.00 & 1.22 & .245 & .000 & 0.95 & -0.08 \\
\hline $\begin{array}{l}\text { Emociones negativas de la } \\
\text { situación social y política }\end{array}$ & 4.00 & 4.25 & 0.88 & .142 & .000 & -1.23 & 1.56 \\
\hline Tristeza-Pena & 3.77 & 4.00 & 1.13 & .224 & .000 & -0.67 & -0.38 \\
\hline Ira & 4.02 & 4.00 & 1.05 & .244 & .000 & -1.04 & 0.56 \\
\hline Indignación & 4.39 & 5.00 & 0.95 & .325 & .000 & -2.05 & 4.51 \\
\hline Vergüenza & 3.83 & 4.00 & 1.27 & .241 & .000 & -0.90 & -0.30 \\
\hline Participación política juvenil & 1.86 & 1.73 & 0.57 & .113 & .000 & 0.89 & 0.89 \\
\hline $\begin{array}{l}\text { Participación política } \\
\text { expresiva-cívica }\end{array}$ & 1.99 & 1.90 & 0.62 & .181 & .000 & 0.68 & 0.19 \\
\hline $\begin{array}{l}\text { Participación política } \\
\text { contenciosa }\end{array}$ & 1.59 & 1.40 & 0.64 & .083 & .027 & 1.37 & 1.94 \\
\hline
\end{tabular}




\section{Relaciones entre variables psicosociales y participación política no convencional}

Se presenta las correlaciones entre las variables sociocognitivas, actitudinales y emocionales asociadas a PPNC. Como se observa en la Tabla 6, según el criterio de Cohen (1988), el Interés en Política presenta una asociación grande con Eficacia Política Interna, y una correlación de magnitud mediana con Eficacia Política Externa; mientras tanto, correlaciona inversamente con Cinismo Político. De igual modo, la Confianza Política presentan una baja asociación directa con la Eficacia Política Interna, pero una correlación mediana con la Eficacia Política Externa. Sobre el Cinismo Político, este luce una correlación inversa y mediana con Eficacia Política Interna.

En caso de las correlaciones con las emociones de la situación política y social, las Emociones Positivas presentan una asociación directa de mediana magnitud con Interés en Política, Eficacia Política Interna y Externa, y con Confianza Política. Solo las Emociones Positivas presentan una correlación inversa y mediana con Cinismo político. En caso de las Emociones Negativas, únicamente se asocia de manera inversa con la Confianza Política y Emociones Positivas.

Respecto a la PP, según la Tabla 6, la PP Expresiva-Cívica presenta asociaciones directas de mediana magnitud con Interés en Política y Eficacia Política Interna; a su vez, una correlación pequeña con Eficacia Política Externa y las Emociones Positivas, y una inversa con Cinismo Político. En caso de la PP Contenciosa, los resultados muestran solo correlaciones directas y pequeñas con Interés en Política, Eficacia Política Interna y Emociones Positivas. 


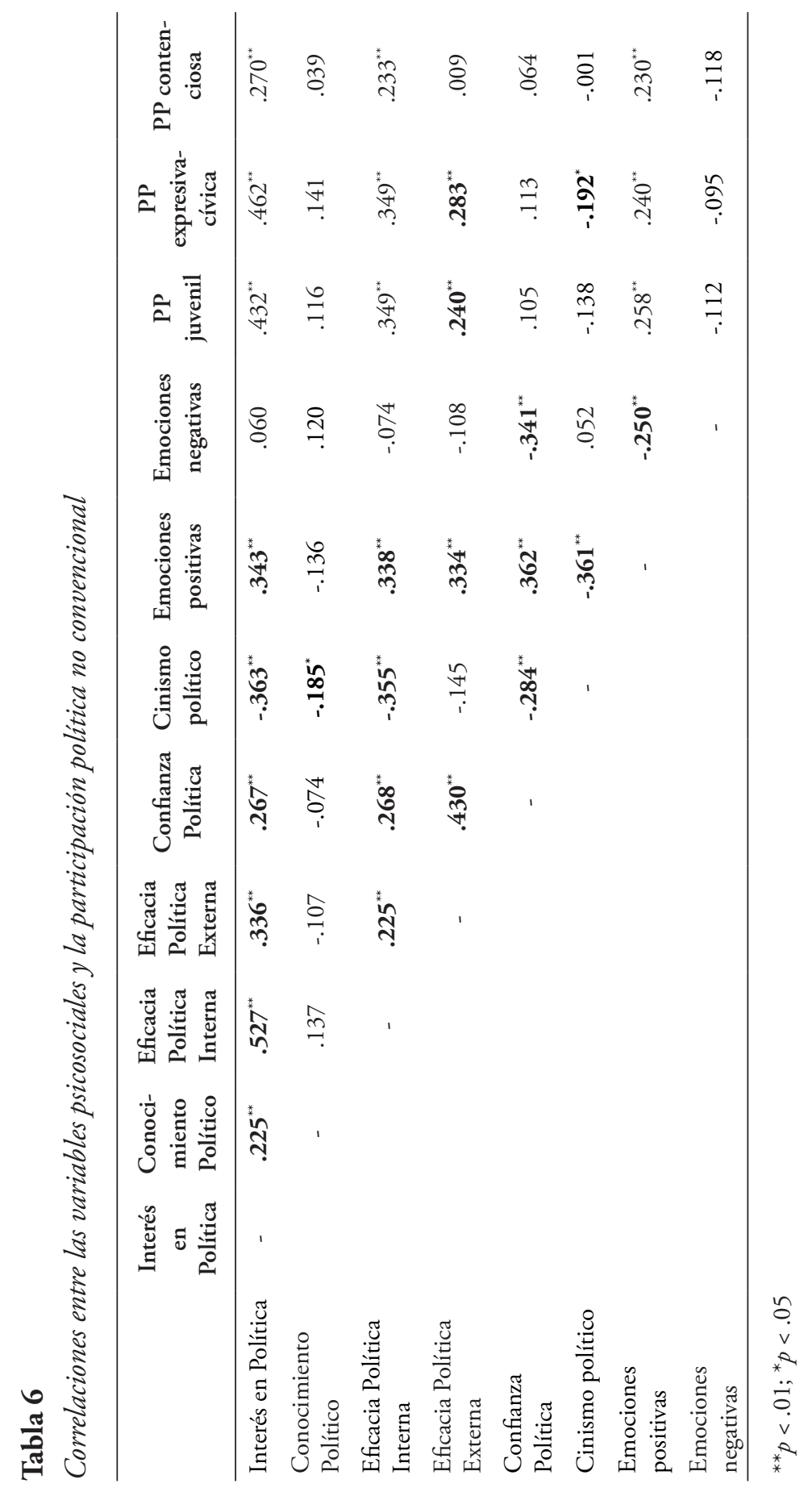


Con el propósito de analizar las variables de manera integrada, se realizó un análisis de caminos tomando en consideración la literatura sobre las relaciones entre las variables socio-cognitivas, actitudinales y la PPNC (Brussino et al., 2011; Jorge, 2016; Mannarini et al, 2008; Páez et al., 2013; Sorribas, 2015; Sorribas \& Brussino, 2013a; 2013b; 2017; Robinson, 2014; Vázquez et al., 2006). Cabe resaltar que no hay un consenso respecto a un modelo integral de cada uno de los factores asociados a la PPNC, por ello, se propone una modelo hipotético (ver Figura 4), teniendo en cuenta los resultados correlacionales y la evidencia de investigaciones previas. No obstante, el modelo hipotético no logró indicadores de ajuste adecuado (ver Tabla 7), y un estimador no fue significativo sobre la relación entre Emociones Positivas, e Interés en Política. En vista de lo anterior, se propone un modelo alternativo (ver Figura 5) que cumple con los criterios de ajuste y de relación óptima entre las variables (ver Tabla 7).

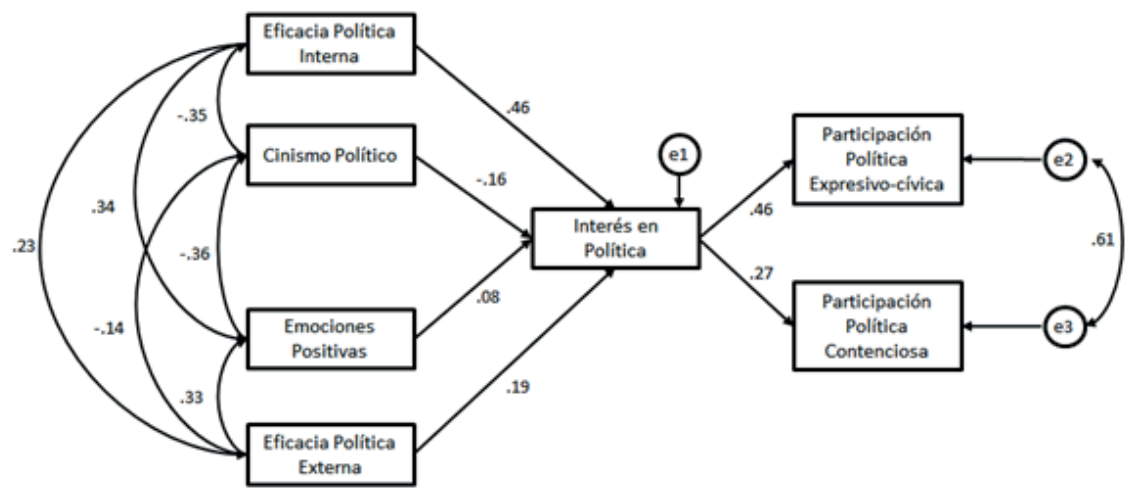

Figura 4. Modelo hipotético de análisis de caminos de la Participación Política Cívico-Expresiva y Contenciosa 


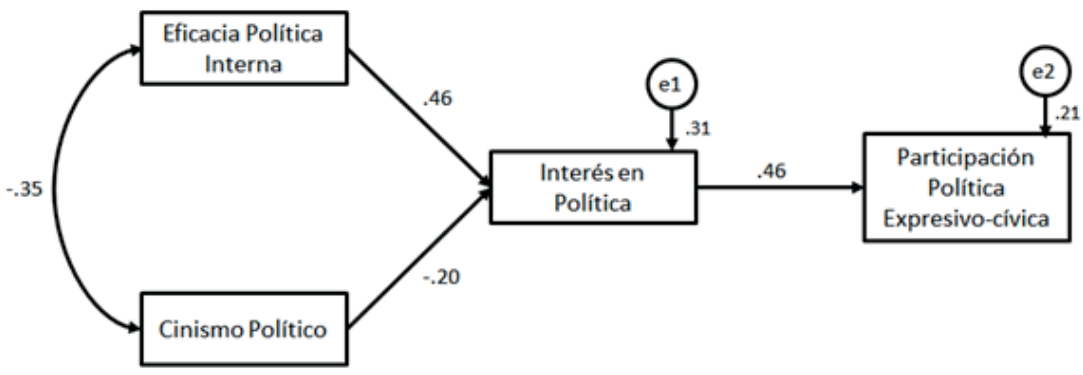

Figura 5. Modelo alternativo de análisis de caminos de la Participación Política Cívico-Expresiva

\section{Tabla 7}

Índices de bondad de ajuste de los análisis de caminos

\begin{tabular}{lcccc}
\hline & $\chi^{2} / \mathrm{gl}$ & CFI & NFI & RMSEA \\
\hline Modelo hipotético & 16.7 & .960 & .930 & .090 \\
Modelo alternativo & 3.45 & .987 & .966 & .074 \\
\hline
\end{tabular}

\section{Discusión Integrada}

\section{Estilos de participación de los jóvenes limeños y cultura política}

Acorde a los distintos modelos de Participación Política (Brussino et al., 2013; Torero et al., 2008), los resultados demuestran que la PP de los jóvenes limeños posee una naturaleza multidimensional. A nivel cualitativo, se identifica tres modalidades orientadas a: (1) incidencia en autoridades, (2) incidencia hacia la sociedad, y (3) expresión. Las dos primeras canalizan el deseo de participar como agentes políticos en busca de cambios en la sociedad, o a un sector de ella. En la misma línea, los resultados cuantitativos permiten distinguir dos estilos de PP 
no convencional, originadas desde su rol como ciudadanos. El primer estilo, catalogado PP Contenciosa, está integrado por acciones de organización e incidencia directa sobre el espacio público físico. Bajo una lógica de confrontación, sus repertorios se orientan a irrumpir la rutina establecida de la dinámica de la sociedad, usualmente como crítica a las decisiones de autoridades o responsables políticos, que se ejemplifica en los reactivos de "Asistir a marchas y/o protestas" y "Toma de oficinas o locales públicos". Esta dimensión guarda sentido con otras tipologías de PP en Latinoamérica (Delfino \& Zubieta, 2010; Sorribas \& Brussino, 2017) y se vincula con las formas más recurrente de PP del primer estudio. Esta forma de PP juvenil se orienta a vigilar e influir en las acciones de las autoridades, quienes representan el orden normativo legal, y cuyas decisiones tienen consecuencias en la calidad de vida y los derechos ciudadanos, como sucedió con las marchas en el Perú en los últimos 5 años (La República, 2016; Perú 21, 2015; El Comercio, 2017).

Los resultados también, delinean otro estilo de PP denominado PP Expresiva-Cívica. Este alude al conjunto de acciones cuyo objetivo es la visibilización de una problemática social o de interés público; también, puede ser considerada como las formas correctas y normativas para desenvolverse como "buenos" ciudadanos en la sociedad. Al contrario del primer estilo de PP, en este estilo emergen comportamientos participativos que expresan un sentido de responsabilidad con los asuntos de interés nacional y local, sin la necesidad de alterar el orden público establecido. Esta propuesta se asemeja al hallazgo de Sandoval y Hatibovic (2018) que confirma tendencias de acción política orientadas a la norma, la legalidad, y la institucionalidad del sistema. Por tanto, no resulta extraño que las conductas de participar en campañas de sensibilización y emisión de pronunciamientos por redes compartan un mismo factor, pues, más allá del canal de expresión (offline u online), dichas acciones políticas comparten un carácter de compromiso cívico y ciudadano, los cuales son repertorios en auge en Latinoamérica (Cubides et al., 2015). 
Cuando se comparan los niveles de ambas modalidades de PP, los jóvenes evidencian una preferencia por acciones cívicas orientadas a la expresión de sus intereses y preocupaciones por asuntos públicos. Esta predilección por la PP cívica-expresiva se podría explicar por dos aspectos: el contexto de desarrollo y socialización política de los jóvenes limeños, y la memoria sobre los procesos políticos del Perú.

Por un lado, el desarrollo social de los jóvenes limeños de 18 a 25 años en un contexto de globalización implica un proceso de intercambio de información social marcado por la inmediatez y agilidad de las tecnologías y plataformas virtuales que han cambiado la manera en que los jóvenes conocen y comprenden la realidad social y política de su sociedad. Para ellos, las redes sociales resultarían ser un mecanismo de control y acceso a información de contenido político que, en tanto respondan a sus intereses personales, serviría como un espacio de relación más horizontal con actores de la sociedad civil, motivándolos a realizar actos de apoyo a causas cívicas y a organizaciones no gubernamentales, a su vez, resulta ser una actividad que menos recursos, costos y no genera desgaste personal (Lilleker \& Koc-Michalska, 2017). Desde los resultados cualitativos, la PP cívica-expresiva sería preferida, no sólo por la accesibilidad de las redes sociales digitales y el menor costo personal, sino porque les permitiría de manera instantánea manifestar su opinión, canalizar su indignación, validar sus convicciones morales, así como proporcionar un entorno más seguro y de fácil intercambio con otros con quienes tienen intereses en común.

Por otro lado, la menor preferencia por PP contenciosa, en tanto estas acciones tienden a alterar el orden público, puede explicarse por su asociación a la memoria sobre la violencia política durante los 80 s y 90s (Rosas, 2009). Incluso en esta época de postconflicto, quienes participan en acciones de protesta serían asociados a grupos de radicalismo político (por ejemplo, MOVADEF), reforzando los estereotipos de jóvenes violentos, así como actitudes de rechazo y repudio social en la ciudadanía en general (Gamarra, 2012; Jave et al., 2015).

Adicionalmente, el nivel de PP de los encuestados podría explicarse en base a las diferentes sub-culturas políticas en Lima, identificadas en 
el estudio de Chaparro (2018). Así, los medianos niveles de sofisticación política, de eficacia política, así como el alto cinismo político, y desconfianza hacia las autoridades, y la alta intensidad emocional negativa hacia la situación del país, indicarían que la muestra podría tratarse de un grupo de jóvenes limeños renegados e insatisfechos con la democracia del país (Chaparro, 2018). Desde esta lectura, los participantes corresponderían a un segmento poblacional que creen poco en la PP convencional y que tampoco tiene expectativas positivas sobre los actos de protestas; más bien, parece que solo se involucran eventualmente en la política cuando afectan sus intereses personales; tal como sucedió con la reforma laboral juvenil (Perú 21,2015). Pese a que este perfil de jóvenes participa en alguna organización civil, la noción de colectividad no gira en torno al nosotros, sino que sería un medio para la PP individual. Por ello, como sugieren los resultados cualitativos, la organización civil no es necesariamente un fin en sí mismo para los jóvenes, como sucedía en los 70s (Rosas, 2009), cuando la militancia guiaba sus ideales sociales y era considerado el principal medio de PP. Entonces, los participantes buscarían los medios adecuados para expresar su descontento hacia el sistema, y re-conectarse con una manera menos tradicional y más horizontal de hacer la política (Nureńa, 2015), ergo optarían por los repertorios orientados al compromiso cívico y visibilización de problemáticas (ambientalistas, de género, barriales, etc.) que, usualmente los interpela personalmente.

\section{La sinergia de los factores psicosociales de la PPNC}

Los resultados revelan relaciones entre algunas medidas sociocognitivas y actitudinales hacia las autoridades y el sistema político. Se evidencia que el cinismo político y la confianza política guardan relación con los niveles de eficacia política interna. Si bien la literatura previa indica que cada una de las tres variables tiene relación directa con la intención de participar y la PP efectiva (Brussino et al., 2009; Páez et al., 2013; Mannarini et al., 2008; Robinson, 2014; Sohl, 2014; Sorribas \& Brussino, 2013a, 2017), también permitiría dar una explicación sobre la relación entre ellas. 
La asociación directa entre confianza política y eficacia política interna puede ser explicada desde la lectura de Bargsted y Torcal (2013). En esa línea, el sentido de autoeficacia para actuar en materia política podría estar afectado por la percepción del contexto social y político, pues un ambiente de inseguridad y desconfianza, cognitivamente, no permitiría a los participantes a evaluar con certeza si sus capacidades y recursos son suficientes para responder a las demandas políticas. De igual modo, los resultados demuestran que el cinismo político se relaciona con los niveles de eficacia política interna. Esto podría explicarse debido a que el alto cinismo político deriva en una percepción de que el cambio en la sociedad sería imposible (Caprara et al., 2009). Si se anula la noción central de cambio de la PP, hallado en el primer estudio, sumado a un sistema político peruano con poca capacidad para hacer frente a la corrupción (Quiróz, 2013), los jóvenes terminarían por creer que cualquier iniciativa individual no sería suficiente para mejorar su calidad de vida, tampoco creerían que su propia capacidad va a generar los cambios deseados en el ámbito político, reduciendo su sentido de eficacia y PP.

Como se observa en los análisis de senderos, en la muestra se evidencia que la eficacia política y el interés en política tienen incidencia sobre la PP efectiva de los jóvenes, en específico, permitiría explicar la PP cívica-expresiva. Dicha relación guarda coherencia con la evidencia previamente documentada con población joven en Perú (Jorge, 2016), Argentina (Brussino et al., 2009; Sorribas \& Brussino, 2013a; 2013b; 2017), España (Páez et al., 2013; Vázquez et al., 2006) e Italia (Mannarini et al., 2008; 2009; Manganelli, Lucidi \& Alivernini, 2014), que reportaron resultados en este mismo sentido.

Al respecto, a partir de la teoría de la cognición social, las personas necesitan dar razones sobre los acontecimientos sociales que viven, y los sucesos políticos no escapan de esa lógica de atribución causal (Sandoval, 2001); por ello, si se realizan atribuciones internas de la actualidad peruana, los participantes pueden realizar conductas de PP que les permita mayor control y despliegue de sus propias capacidades. Asimismo, se demuestra que la curiosidad por asuntos públicos 
funcionaría como base motivacional de la PP (Miller, 2011; Sorribas \& Brussino, 2013a), aumentando sus niveles de PP cívica-expresiva; en caso del presente estudio, en la medida en que los participantes se informan de distintos sucesos políticos afines a sus intereses personales, más aún si dichos intereses guardan relación con inquietudes identitarias en temas de diversidad sexual, género, estilos de consumo, y valores de inclusión y justicia.

Además, el efecto de la eficacia política interna sobre la PP cívicaexpresiva está mediado por el grado de curiosidad y atención sobre los asuntos políticos; resultado coincidente con los hallazgos de Prior (2018) y Reichert (2018). Lo anterior no quiere decir que los niveles de autoeficacia política no tengan incidencia en la PP de corte cívico y expresivo, sino que la probabilidad de que los encuestados se involucren en PP se incrementaría cuando desarrollan un sustrato motivacional que los mantenga atraídos hacia los sucesos políticos. Así, si las expectativas de generar un cambio, paulatinamente van siendo frustradas y no se traducen en logros reales, simbólicos o percibidos, el interés por los asuntos públicos se reduciría, terminando por tener menos motivos para involucrarse en algún repertorio de PP. Esta dinámica de relacionamiento entre las variables se acentúa más en la población juvenil, pues son el segmento poblacional marcado por la desconfianza hacia quienes representan al sistema democrático (Arias \& Alvarado, 2015; Nureña, 2015).

Ahora bien, al igual que otros estudios (Alberaci et al., 2012; Páez et al., 2013; Rico et al., 2017; Sabucedo \& Vilas, 2014), los resultados indican que otro factor asociado a la PP son las emociones, en este estudio, serían las emociones positivas hacia la situación social y política. Este hallazgo podría explicarse dado el rol de las emociones de optimismo y esperanza sobre el comportamiento político (Vilas et al., 2016), pues estas emociones estimularían, en caso de los encuestados, un correlato cognitivo de que el futuro que cada uno desea es posible, reforzando la motivación para tomar acciones a largo plazo y soportar las dificultades derivadas de la PP. Además, en el marco de un sistema político percibido como corrupto, débil, y carente de confianza (IOP- 
PUCP, 2017), estas emociones positivas serían relevantes para enfrentar la situación política descrita, en la medida en que podrían dotar de significado y sentido a sus formas personales de PP. Así, cualquier propuesta de acción sobre el objeto de evaluación emocional, en este caso, la situación social y política, desarrollaría en los encuestados la seguridad necesaria para transformar su intención en acciones efectivas.

En caso de las emociones negativas, contrario a lo esperado, los resultados señalan que no existe relación entre ellas y la PP. A pesar de los altos niveles de emocionalidad personal negativa hacia la situación del país, estas no tienen efecto sobre la PP. Esto podría explicarse por la cualidad del objeto de evaluación emocional, pues la literatura revisada sobre emociones y PP en protestas y movimientos sociales (Rico et al., 2017; van Zomeren et al., 2008; Wlodarczyk et al., 2017), usualmente coloca como objeto de evaluación emocional a eventos específicos, desde atentados, conflictos armados, o acciones concretas de las autoridades, mientras que, en caso del estudio 2, refiere a un objeto general y amplio. Desde los hallazgos del estudio 1 , si bien se señala que la indignación funcionaría como antecedente de PP, esta emoción negativa no actúa sola, sino en conjunto con variables interpersonales de identificación y soporte social. Por tanto, el involucramiento en la PP, mediante emociones negativas, podría estar afectado por la falta de especificidad de un objeto que canalice la reacción emocional (p. e. responsables políticos); o bien, por variables interpersonales que no se tomaron en cuenta en el estudio 2. Otra explicación tentativa podría deberse a que la creciente devaluación del funcionamiento del sistema democrático en el Perú (Carrión et al., 2018), frenaría la reacción emocional negativa de los jóvenes para iniciar o ser parte de alguna forma de PP.

En conclusión, ambos estudios delinean el carácter multidimensional de la PP no convencional en una muestra de jóvenes limeños, con preferencia por modalidades expresivas y cívicas. La PP les permite canalizar sus emociones positivas, satisfacer la necesidad de relación, y adquirir habilidades valoradas social y culturalmente que, en consecuencia, dialogan con su proyecto de desarrollo como ciudadanos. Si bien la concepción de PP se orienta a la búsqueda de un cambio, 
a nivel macro o micro, los motivos de PP tienden a regirse bajo una lógica individual de costo y beneficio, que les permita una expansión de sus capacidades personales.

A futuro, sería importante evaluar la PP diferenciada entre acciones en redes sociales y voluntariados, así como incorporar medidas de variables interpersonales que puedan afectar el efecto de las variables actitudinales e intrapersonales sobre la PP de los jóvenes. De igual modo, se sugiere indagar en grupos de PP de corte más conservador, con fin de identificar sus motivos y atribuciones de PP.

\section{Referencias}

Aguilera, O. (2010). Acción colectiva juvenil: de movidas y finalidades de adscripción. Nómadas, 32, 81-97.

Alberici, A. I., Milesi, P., Malfermo, P., Canfora, R. \& Marzana, D. (2012). Comparing social movement and political party activism: the psychosocial predictors of collective action and the role of the Internet. TAISP Conference. Canterbury: University of Kent.

Alvarado, S. V., Ospina-Alvarado, M. C. \& García, C. M. (2012). La subjetividad política y la socialización política, desde las márgenes de la psicología política. Revista Latinoamericana de Ciencias Sociales, Niñez, y Juventud, 10(1), 235-256.

Ames, M. (2013). Engaging "apolitical” adolescents: Analyzing the popularity and educational potential of dystopian literature post-9/11. The High School Journal, 97(1), 3-20.

American Psychological Association (APA, 2017). Ethical principles of psychologist and code of conduct.

Arias-Cardona, A. M. \& Alvarado, S. V. (2015). Jóvenes y política: de la participación formal a la movilización informal. Revista Latinoamericana de Ciencias Sociales, Niñez y Juventud, 13(2), 581-594. 
Barnes, S. H. \& Kasse, M. (1979). Political action: mass participation in five western democracies. California, Estados Unidos de América: SAGE Publications.

Barrett, L. F., Mesquita, B, Ochsner, K. N. \& Gross, J. J. (2007). The experience of emotion. The Annual Review of Psychology, 58, 373-403.

Bargsted, M. \& Torcal, M. (2013). Explicando la confianza política ¿economía o política? Un análisis longitudinal y comparado de Europa y América Latina. En L. Paramio (Coord.), Desafección politica y gobernabilidad: el reto politico (pp. 201-228). España: Marcial Pons.

Bélanger, E. \& Aarts, K. (2006). Explaining the rise of the LPF: Issues, discontent and the 2002 Ducth election. Acta Politica, 41, 4-20. Beramendi, M. \& Zubieta, E. (2013). Norma perversa: trasgresión como modelo de legitimidad. Universitas PSychologica, 12(2), 591-600.

Bericat Alastuey, E. (2002). ¿Sienten las sociedades? Emociones individuales, sociales y colectivas. En P. Fernández \& N. Ramos, Corazones Inteligentes. Barcelona: Editorial Cairos.

Berreota, H. \& Sandoval, J. (2014). Protestas, participación y educación pública: discurso sobre lo público en las movilizaciones estudiantiles en Chile. Educar em Revista, 53, 19-38.

Botero, P., Torres, J. \& Alvarado, S. V. (2008). Perspectivas teóricas para comprender la categoría participación ciudadana-política juvenil en Colombia. Revista Latinoamericana de Ciencias Sociales, Niñez y Juventud, 6(2), 565-611.

Braun, V. \& Clarke, V. (2006). Using thematic analysis in psychology. Qualitative Research in Psychology, 3(2). https://doi. org/10.1191/1478088706qp063oa

Brussino, S., Medrano, L., Sorribas, P. \& Rabbia, H. H. (2011). Young adults' knowledge of politics: Evaluating the role of socio-cognitive variables using structural equations. The Spanish Journal of Psychology, 14(1), 183-194. https://doi.org/10.5209/ rev_SJOP.2011.v14.n1.16 
Brussino, S., Rabbia, H. H. \& Sorribas, P. (2009). Perfiles sociocognitivos de la participación política de los jóvenes. Interamerican Journal of Psychology, 43(2), 279-287.

Brussino, S., Sorribas, P., Gutiérrez, J., Imhoff, D., Kerman, L., Medrano, L., Ricci, P., Spinuzza, F. \& Vidal, N. (2006). Participación politica en jóvenes: un análisis descriptivo. XIII Jornadas de Investigación y Segundo Encuentro de Investigadores en Psicología del Mercosur. Facultad de Psicología - Universidad de Buenos Aires, Buenos Aires.

Caprara, G. V., Vecchione, M., Capanna, C. \& Mebane, M. (2009). Perceived political self-efficacy: theorym assessment, and applications. European Journal of Social Psychology, 1002-1020. doi: 10.1002/ejsp.604

Carrión, J., Zárate, P., Boidi, F. \& Zechmeister, E. (2018). Cultura Política de la Democracia en Perú y las Américas. 2016/2017. Lima, Perú: IEP y LAPOP.

Chaparro, H. (2018). Afectos y desafectos: Las diversas subculturas politicas en Lima. Lima, Perú: Instituto de Estudios Peruanos.

Chóliz, M. (2005). Psicología de la emoción: el proceso emocional. Recuperado de https://www.uv.es/

Ciarrochi, J., Parker, P., Kashdan, T. B., Heaven, P. C. L. \& Barkus, E. (2015). Hope and emotional well-being: A six-year study to distinguish antecedents, correlates, and consequences. The Journal of Positive Psychology, 1-13. https://doi.org/10.1080 /17439760.2015.1015154

Clarke, V. \& Braun, V. (2013). Teaching thematic analysis: Overcoming challenges and developing strategies for effective learning. The Psychologist, 26(2), 120-123.

Conge, P. J. (1988). The concept of political participation: Toward a definition. Comparative Politics, 20(2), 241-249.

Córdova, C. (2013). Autoritarismo, actitudes politicas y participación politica no convencional (Tesis de Licenciatura). Repositorio de la Pontificia Universidad Católica del Perú. 
Cubides, H., Borelli, S., Unda, R. \& Vázquez, M. (2015). Juventudes Latinoamericanas: Prácticas Socioculturales, Politicas y Politicas Públicas. Bueno Aires, Argentina: CLACSO.

Cueto, R. M., Fourment, K., Seminario, E. \& Fernández, A. (2014). Orientación a la dominancia y representaciones sociales de Estado, Mercado y política en estudiantes universitarios de Lima, Perú. En M. Rodríguez \& G. Grondona (Coords.), Juventudes y Política: cambios sociopoliticos en América del Sur (pp. 99-122). Quito, Ecuador: Universidad Politécnica Salesiana.

DiGrazia, J. (2014). Individual protest participation in the United State: conventional and unconventional activism. Social Science Quarterly, 95(1), 111-131.

Delfino, G. \& Zubieta, E. M. (2014). Participación política pacífica y agresiva: Aprobación y eficacia atribuida. Psicología Política, 48, $25-46$.

Delfino, G. \& Zubieta, E. M. (2010). Participación política: Concepto y modalidades. Anuario de Investigaciones, 17, 211-220.

Delfino, G., Zubieta, E. M. \& Muratori, M. (2013). Tipos de participación política: Análisis factorial confirmatorio con estudiantes universitarios de Buenos Aires, Argentina. Psicología Politica, 13(27), 301-318.

De Rivera, J. (1992). Emotional climate: social structure and emotional dynamics. International Review of Studies on Emotion, 2, $197-218$.

Del Tronco, J. (2012). Las causas de la desconfianza política en México. Perfiles Latinoamericanos, 40, 227-251.

Diekman, A. \& Schneider, M. C. (2010). A social role theory perspective on genders gaps in political attitudes. Psychology of Women Quarterly, 34, 486-497.

Domínguez, L. (1989). Nacionalismo y militarismo en el Perú: 1968-1980. Omnia, 16. Recuperado de http://www.posgrado. unam.mx/

Domínguez, F., López, R. \& Ortiz-Henderson, G. (2017). Redes sociales digitales y participación política de jóvenes universitarios 
de México y Chile: una revisión de estudios. Revista Latinoamericana de Ciencias de la Comunicación, 14(26), 87-95.

Edelman (2019). 2019 Edelman Trust Barometer. Global Report.

ElComercio(11 denoviembrede2017). Niunamenos: convocanamarcha para el 25 de noviembre. El Comercio. Recuperado de: https:// elcomercio.pe/peru/realizo-marcha-pais-noticia-476727-noticia Eisinger, R. (2000). Questioning cynicism. Culture and Society, 3, 55-60.

Espinosa, A., Cueto, R. M. \& Schmitz, M. (2012). De una Psicología Social de fenómenos políticos al desarrollo de la Psicología Política en el Perú. Psicología Politica, 12(25), 465-479.

Gamarra, J. (2012). MOVADEF: radicalismo político y relaciones intergeneracionales. Revista Argumentos, 6(5), 1-6.

Gasca-Pliego, E. \& Olvera-García, J. C. (2011). Construir ciudadanía desde las universidades, responsabilidad social universitaria y desafíos ante el siglo XXI. Convergencia Revista de Ciencias Sociales, 18(56), 37-58.

Gil de Zúñiga, H., Molyneux, L. \& Zheng, P. (2014). Social media, political expression, and political participation: Panel analysis of lagged and concurrent relationships. Journal of Communication, 64, 612-634. https://doi.org/10.1111/jcom.12103

Gil de Zúniga. H. \& Valenzuela, S. (2012). The mediating path to stronger citizenship: Online and offline networks, weak ties, and civic engagement. Communication Research, 38(3), 397-421. https://doi.org/10.1177/0093650210384984

Goodwin, C. J. (2010). Ethics in psychological research. En C.J. Goodwin (Ed.), Research in Psychology Methods and Design Sixth Edition, (pp. 39-80). Estados Unidos: Wiley.

Grompone, R., y Tanaka, M. (2009). Entre el crecimiento económico y la insatisfacción social: las protestas sociales en el Perú actual. Lima: IEP.

Grönlund,K.\&Milner,H.(2006).Thedeterminantsofpoliticalknowledge in comparative perspective. Scandinavian Political Studies, 29, 386-406. https://doi.org/10.1111/j.1467-9477.2006.00157.x 
Hardin, R. (2000). Do we want trust in government? En M.E. Warren (Ed.), Democracy and Trust., (pp. 22-41). Cambridge: Cambridge University Press.

Hooghe, M. \& Zmerli, S. (2013). Introduction: the Context of Political Trust. En S. Zmerli y M. Hooghe (Eds.), Political Trust: Why Context Matters (pp. 1-11). Colchester: ECOR Press.

Instituto de Opinión Pública (IOP-PUCP, 2017). Apoyo a la democracia, confianza en el congreso y en los partidos políticos. Boletín $N^{\circ} 145$ Estado de la Opinión Pública.

IPSOS (1 de octubre del 2017). ¿En qué instituciones confían los peruanos? El Comercio.

Imhoff, D. \& Brussino, S. (2016). Socialización política: La dialéctica relación entre individuo y sociedad. En Brussino, S. (Coord.), Politicamente Contribuciones desde la Psicología Politica en Argentina (pp. 37-70). Córdoba, Argentina: CIECS.

Janos, E., Espinosa, A. \& Pacheco, M. (2018). Bases ideológicas de la percepción del sistema normativo y le cinismo político en adultos de sectores urbanos del Perú. PSYKHE, 27(1), 1-14. https://doi.org/10.7764/psykhe.27.1.1176

Jasper, J. M. (2011). Emotions and social movements: Twenty years of theory and research. Annual Review of Sociology, 37, 285-303. https://doi.org/10.1146/annurev-soc-081309-15001515

Jave, I., Cépeda, M. \& Uchuypoma, D. (2015). La acción política frente al estigma de la violencia entre los jóvenes universitarios posconflicto: los casos de la Universidad Nacional Mayor de San Marcos y la Universidad Nacional de San Cristóbal de Huamanga. Anthropologica, 33(34), 187-202.

John, P., Fieldhouse, E. \& Liu, H. (2011). How civic is the civic culture? Mapping the routes to community participation using 2005 English Citizenship Survey. Political Studies, 59(2), 230-252. https://doi.org/10.1111/j.1467-9248.2011.00891.x

Jorge, A. (2016). Relaciones entre ideología, participación y cinismo politico en jóvenes de Lima (Tesis de Licenciatura). Pontificia Universidad Católica del Perú, Lima. 
Kaase, M. \& Marsh, A. (1979). Political action: A theoretical perspective. En Barnes, S. H. \& Kaase, M. (Eds.), Political action: Mass participation in five western democracies (pp. 27-56). Beverly Hills, CA: Sage.

Klandermans, B. (1984). Mobilization and participation: Socialpsychological expansions of resource mobilization theory. American Sociological Review, 49, 583-600.

Klandermans, B. (2014). Identity politics and politicized identities: Identity process and the dynamics of protest. Political Psychology, 35(1), 1-22.

Kline, R. B. (2011). Principles and practice of structural equation modeling ( $3^{\mathrm{a}} \mathrm{ed}$.). New York, NY: Guilford Press.

Krampen, G. (2000). Transition of adolescent political action orientations to voting behavior in early adulthood in view of social-cognitive action theory model of personality. Political Psychology, 21(2), 277-297.

Krauskopf, D. (2000). Dimensiones críticas en la participación social de las juventudes. En Krauskopf, D. (Ed.), Participación y Desarrollo Social en la Adolescencia (pp. 119-134). Buenos Aires, Argentina: CLACSO.

Latinobarómetro (2017). Informe 2017. Santiago de Chile, Chile.

La República (31 de mayo del 2016). Marcha 'Keiko no va': Así fue la movilización contra la candidatura de Keiko Fujimori. La República. Recuperado de: http://larepublica.pe/ politica/772365-marcha-no-keiko-miles-se-manifiestan-hoyen-el-peru-y-el-mundo

Lilleker, D. \& Koc-Michalska, K. (2017). What drives political participation? Motivations and mobilization in digital age. Political Communication, 34(1), 21-43. https://doi.org/10.108 $0 / 10584609.2016 .1225235$

Lorenzo-Seva, U. \& Ferrando, P. J. (2006). Manual of The Program Factor. Tarragora, Espańa: Universitat Rovira i Virgili.

Lorenzo-Seva, U. \& Ferrando, P. J. (2019). Unrestricted factor analysis of multidimensional test items based on an objectively refined 
target matrix. Behavior Research Methods, 1-15. https://doi. org/10.3758/s13428-019-01209-1

Luskin, R. C. (1990). Explaining political sophistication. Political Behavior, 12(4), 331-361.

Neundorf, A. \& Niemi, R. G. (2014). Editorial Beyond political socialization: New approaches to age, period, cohort analysis. Electoral Studies, 33, 1-6.

Niemi, R. G., Craig, S. \& Mattei, F. (1991). Measuring internal political efficacy in the 1988 National Election Study. The American Political Science Review, 85(4), 1407-1413.

Nowell, L., Norris, J. M., White, D. \& Moules, N. (2017). Thematic analysis: Striving to meet the trustworthiness criteria. International Journal of Qualitative Methods, 16, 1-13. https:// doi.org/10.1177/1609406917733847

Nureña, C. R. (2015). Juventud y cultura politica en el Perú: El caso de los estudiantes de Universidad Nacional Mayor de San Marcos de Lima. (Tesis de Magíster). Recuperado del repositorio de la Universidad Iberoamericana. México D. F.

Manganelli. S., Lucidi, F. \& Alivernini, F. (2014). Adolescents' expected civic participation: The role of civic knowledge and efficacy beliefs. Journal of Adolescence, 37, 632-641. https://doi. org/10.1016/j.adolescence.2014.05.001

Mannarini, T., Legittimo, M. \& Talo, C. (2008). Determinants of social and political participation among youth: a preliminary study. Psicología Politica, 36, 95-117.

Mannarini, T., Roccato, M., Fedi, A. \& Rovere, A. (2009). Six factors fostering protest: Predicting participation in locally unwanted land uses movements. Political Psychology, 30(6), 895-920. https://doi.org/10.1111/j.1467-9221.2009.00732.x

Miller, P. R. (2011). The emotional citizen: emotion as a function of political sophistication. Political Psychology, 32(4), 575-600. https://doi.org/10.1111/j.1467-9221.2011.00824.x 
Ocampo, A. M. (2011). Ciudadanía juvenil, juventud y Estado: discursos de gobierno sobre sus significados. Revista Latinoamericana de Ciencias Sociales, Niñez y Juventud, 1(9), 287-303.

Opdycke, K., Segura, P. \& Vásquez, A. M. (2013). The effects of political cynicism, political information efficacy and media consumption on intended voter participation. Colloquy, 9, 75-97.

Páez, D., Javaloy, F., Wlodarczyk, A., Espelt, E. \& Rimé, B. (2013). El movimiento 15-M: sus acciones como rituales, compartir social, creencias, valores y emociones. Revista de Psicología Social, 28(1), 1-15.

Pattyn, S., Van hiel, A., Dhont, K. \& Onreat, E. (2012). Stripping the political cynicism: a psychological exploration of the concept of political cynicism. European Journal of Personality, 26, 566-579. https://doi.org/10.1002/per.858

Perú 21 (26 de Enero del 2015). Ley Pulpín: Pleno del Congreso derogó régimen laboral juvenil. Perú 21. Recuperado de: https:// peru21.pe/politica/ley-pulpin-pleno-congreso-derogo-regimenlaboral-juvenil-164000

Pfaff, N. (2009). Youth culture as a context of political learning. How young people politicize each other. Young, 17(2), 167-189. https://doi.org/10.1177/110330880901700204

Porter, T. J. (2013). Moral and political identity and civic involvement in adolescents. Journal of Moral Education, 42(2), 239-255. https://doi.org/10.1080/030572 40.2012.761133

Prior, M. (2018) The psychology of political interest. En M. Prior (Ed.), Hooked How Politics Captures Peoples' Interest, (pp. 19-39). New Jersey, Estados Unidos: Cambridge University Press.

Quiroz, A. W. (2013). Historia de la corrupción en el Perú. Lima, Perú: IEP.

Reichert, F. (2018). How important are political interest and internal political efficacy in the prediction of political participation? Longitudinal evidence from Germany. Revista de Psicología Social, 1-42. https://doi.org/10.1080/02134748.2018.1482056 
Rico, D., Alzate, M. \& Sabucedo, J. M. (2017). El papel de la identidad, la eficacia y las emociones positivas en las acciones colectivas de resistencia pacífica en contextos violentos. Revista Latinoamericana de Psicología, 49, 28-35. https://doi.org/10.1016/j. rlp.2015.09.013

Rivera, S. (2019). Confianza y participación política en América Latina. Revista Mexicana de Ciencias Politicas y Sociales Nueva Época, 64(235), 555-584. https://doi.org/10.22201/ fcpys.2448492xe.2019.235.65728.

Robinson, J. G. (2014). Political cynicism and foreclosure crisis. Social Justice, 40(3), 99-118.

Rodríguez, M. A. (2016). La relación entre el conocimiento político y la democracia: una experiencia a partir del Estado de Sinaloa, México. Revista Debates, 10(2), 149-170.

Rodríguez, M., Sabucedo, J. M. \& Costa, M. (1993). Factores motivacional y psicosociales asociados a distintos tipos de acción política. Psicología Política, 7, 19-38.

Rosas, F. (2009). Breve Historia General de los Peruanos. Arequipa, Perú: Ediciones El Lector.

Ryan, R. M. \& Deci, E. L. (2017). Basic psychological needs theory: Satisfaction and frustration of Autonomy, Competence, and Relatedness in relation to Psychological Wellness and Full Functioning. En R. M. Ryan \& E. L. Deci (Eds.), SelfDetermination Theory, (pp. 239-271). New York, Estados Unidos: The Guilford Press.

Sabucedo, J. M. (1988). Participación política. En J. Seone \& A. Rodríguez, Psicología Política (pp. 165-194). Madrid, España: Pirámide.

Sabucedo, J. M., Dono, M., Alzate, M. \& Seoane, G. (2018). The importance of protesters' morals: moral obligation as key variable to understand collective action. Frontiers in Psychology, 9, 1-12. https://doi.org/10.3389/fpsyg.2018.00418 
Sabucedo, J. M., Durán, M. \& Alzate, M. (2010). Identidad colectiva movilizada. Revista de Psicología Social, 25(2), 189-201. https:// doi.org/ 10.1174/021347410791063822

Sabucedo, J. M. \& Vilas, X. (2014). Anger and positive emotions in political protest. Universitas Psychologica, 13(3), 829-838.

Sandoval, M. (2012). La desconfianza de los jóvenes: sustrato del malestar social. Última Década, 36, 43-70.

Sandoval, J. \& Hatibovic, F. (2018). Validación de la escala de tendencias de acción aolítica en una muestra de estudiantes universitarios chilenos. PSYKHE, 27(1), 1-17. https://doi. org/10.7764/psykhe.27.1.1030

Sandoval, S. A. M. (1994). Algumas reflexóes sobre cidadania e formação de consciência política no Brasil. En Spink, M. J. (Ed.), A cidadania em construção: Uma reflexão transdisciplinar, (pp. 59- 73). São Paulo: Cortez.

Sandoval, S. A. M. (2001). The crisis of Brazilian labor movement and the emergence of alternative forms of working-class contentions in 1900s. Revista de Psicología Política, 1(1), 173-195.

Sandoval, S. A. M. \& Silva, A. S. (2016). O modelo de análise da consciência política como contribuicão para a psicologia política dos movimientos sociais. En Uhng, D. \& Lacerda, F. (Eds.), Psicologia, politica e movimentos sociais, (pp. 25- 57). Petrópolis, Brasil: Vozes.

SENAJU (2015). Informe nacional de las juventudes en el Perú 2015. Lima, Perú: Fondo de la Población de la Naciones Unidas.

Sergovia, C. (2016). Desigualdad de Información: Una Exploración de los Antecedentes del Conocimiento Político en Chile. PSYKHE, 25(2), 1-16. https://doi.org/10.7764/psykhe.25.2.847

Simon, B. \& Klandermans, B. (2001). Politicized collective identity: a social psychological analysis. American Psuchologist, 56(4), 319-335.

Sohl, S. (2014). Youths' Political Efficacy: Sources, effects and potentials for political equality. Örebro University. 
Sola, S. \& Hernández, V. (2017). Abstención política y nuevas formas de participación política de los jóvenes: análisis comparativo entre Chile y España. Revista Latina de Comunicación Social, 72, 629-648. https://doi.org/10.4185/RLCS-2017-1183

Somim, I. (2016). Democracy and Political Ignorance: Why smaller goverment es smarter (2nd Edition). Standford: Standford University Press.

Sorribas, P. \& Brussino, S. (2013a). Participación política orientada al sistema representativo: dimensiones y factores explicativos. Psicología Politica, 47, 91-112.

Sorribas, P. \& Brussino, S. (2013b). La participación política contenciosa: desarrollo de un modelo explicativo desde la cognición social. Quaderns de Psicología, 15(2), 7-22.

Sorribas, P. \& Brussino, S. (2017a). Dimensiones y factores explicativos de la participación política: La relevancia del enfoque psicosocial. En Brussino, S. (Coord.), Politicamente Contribuciones desde la Psicología Politica en Argentina (pp. 103-130). Córdoba, Argentina: CIECS.

Sorribas, P. \& Brussino, S. (2017b). Participación política: el aporte discriminante de actitudes ideológicas, valores y variables sociopsicológicas. Revista de Psicología, 35(1), 311-345. https:// doi.org/10.18800/psico.201701.011

Torcal, M., Montero, J. R. \& Teorell, J. (2006). La participación política en Espańa: Modos y niveles en perspectiva comparada. Revista de Estudios Politicos, 132, 7-41.

Valencia, J. F. (1990). La lógica de la acción colectiva: tres modelos de análisis de la participación política no institucional. Revista de Psicología Social, 5(2-3), 185-214.

Uriarte, J. (2005). En la transición a la edad adulta. International Journal of Developmental and Educational Psychology, 3(1), 145-160.

van Deth, J. W. (2001) Studying Political Participation: Towards a Theory of Everything? Joint Sessions of Workshops of the European Consortium for Political Research, Grenoble, France. 
van Deth, J. W. (2014). A conceptual map of political participation. Acta Politica, 49(3), 349-367. https://doi.org/10.1057/ ap. 2014.6

van Stekelenburg, J. (2013). Moral Iniciatives. En David A. Snow, Donatella della Porta, Bert Klandermans, and Doug McAdam (Eds.), The Wiley-Blackwell Encyclopedia of Social and Political Movements. doi: 10.1002/9781405198431.wbespm 132

van Stekelenburg, J. \& Klandermans, B. (2010). The social psychology of protest. Current Sociology Review, 61(5-6), 886-905. https:// doi.org/10.1177/0011392113479314

van Stekelenburg, J., Klandermans, B. \& van Dijk, W. W. (2009). Context matters: Explaining how and why mobilizing context influences motivational dynamics. Journal of Social Issues, 65(4), 815-838.

van Stekelenburg, J., Klandermans, B. \& van Dijk, W. W: (2011). Combining motivations and emotion: The motivational dynamics of protest participation. Revista de Psicología Social, 26(1), 91-104. https://doi.org/10.1174/021347411794078426

van Zomeren, M., Postmes, T. \& Spears, R. (2008). Toward an integrative social identity model of collective action: A quantitative research synthesis of three socio-psychological perspectives. Psychological Bulletin, 134, 504-535. https://doi. org/10.1037/0033-2909.134.4.504

van Zomeren, M., Wayne, C. \& Spears, R. (2010). Does group efficacy increase group identification? Resolving their paradoxical relationship. Journal of Experimental Social Psychology, 46(6), 1055-1060. https://doi.org/10.1016/j.jesp.2010.05.006

Vázquez, J. J., Panadero, S. \& Rincón, P. P. (2006). Acción política no convencional en universitarios espańoles, chilenos, salvadoreños y nicaragüenses. Psicología Politica, 33, 25-41.

Venturo, S. (2001). Contrajuventud: Ensayos sobre juventud y participación politica. Lima, Perú: IEP Ediciones.

Vilas, X., Alzate, M. \& Sabucedo, J. M. (2016). Mobilized citizenchip: motives, emotions and context. Diversitas: Perspectivas 
en Psicología, 12(2), 167-181. https://doi.org/10.15332/ s1794-9998.2016.0002.01

Vilas, X. \& Sabucedo, J. M. (2012). Moral obligation: A forgotten dimension in the analysis of collective action. Revista de Psicología Social, 27(3), 369-375. https://doi. org/10.1174/021347412802845577

Wlodarczyk, A., Besabe, N., Páez, D. \& Zumeta, L. (2017). Hope and anger as mediators between collective actions frames and participation in collective mobilization: The case of $15-\mathrm{M}$. Journal of Social and Political Psychology, 5(1), 200-223. https:// doi.org/10.5964/jspp.v5i1.471

Youniss, J. (2009). When morality meets politics in development. Journal of Moral Education, 38(2), 129-144. https://doi. org/10.1080/03057240902792660

Zimmerman, M. A. \& Zahniser, J. H. (1991). Refinements of sphere-specific measures of perceived control: Development of sociopolitical control scale. Journal of Community Psychology, 19, 189-104.

Recibido: 2020-02-25

Revisado: 2021-03-12 Aceptado: 2021-04-08 


\section{Apéndices}

Apéndice A: Consentimiento Informado - Estudio Cualitativo

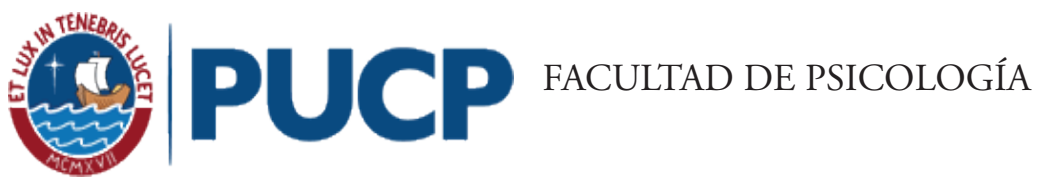

\section{CONSENTIMIENTO INFORMADO}

En marco del estudio de "Los Factores Psicosociales de la Participación Política No Convencional en jóvenes-universitarios-en la ciudad de Lima", invito a que acepte de manera voluntaria a participar en el proceso de dicha investigación, llevado a cabo por el Lic. Meir Tintaya Orihuela.

Por tal motivo, le solicitamos colaborar con una entrevista dirigida a jóvenes mayores de 18 ańos y residentes en Lima, la cual busca indagar en las experiencias de participación. Cabe mencionar que la entrevista tiene una duración aproximadamente de 30 minutos, y será grabada para facilitar el análisis de información. Con el fin de salvaguardar la confidencialidad del entrevistado(a), todo manejo de la información será codificada bajo un seudónimo. Si en alguna pregunta le resulta incómoda, tiene la libertad de no responderla, incluso, dar culminada la entrevista. Recuerde que no existe respuestas correctas o incorrectas, más bien, toda opinión resulta valiosa para el estudio.

De antemano, agradecemos su colaboración y ante cualquier duda, puede comunicarse al siguiente correo: mtintaya@pucp.pe.

Firma de la participante

Firma del investigador

Lima, de del 2018 
Apéndice B: Consentimiento Informado - Estudio Cuantitativo

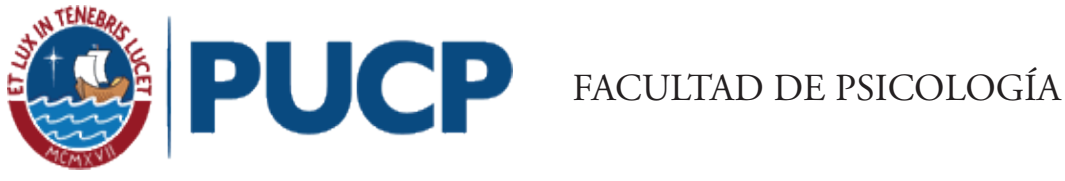

\section{CONSENTIMIENTO INFORMADO}

En marco del estudio de "Los Factores Psicosociales de la Participación Política No Convencional en jóvenes en la ciudad de Lima", invito a que acepte de manera voluntaria a participar en el proceso de dicha investigación, llevado a cabo por el Lic. Meir Tintaya Orihuela.

Por tal motivo, le solicitamos colaborar con el llenado de un cuestionario dirigido a jóvenes mayores de 18 ańos y residentes en Lima, la cual busca conocer su opinión sobre las razones y experiencias de participación política y ciudadana de jóvenes en Lima. Además, el cuestionario tiene una duración aproximadamente de 20 minutos. Con el fin de salvaguardar la confidencialidad del entrevistado(a), todo manejo de la información será recopilada bajo un código, y los análisis de información serán de manera grupal, de modo que no será identificado. Si en alguna pregunta le resulta incómoda, tiene la libertad de no responderla, incluso, dar culminado el cuestionario si lo considera necesario. Recuerde que no existe respuestas correctas o incorrectas, más bien, toda respuesta resulta valiosa para el estudio.

De antemano, agradecemos su colaboración y, para cualquier información adicional y/o dificultad, el participante puede contactarse al siguiente correo:mtintaya@pucp.pe.

Firma de la participante

Firma del investigador

Lima, de del 2018 


\section{Apéndice C: Guía de Entrevista}

\section{Bloque Inicio:}

1. En general, cuéntame acerca de ti, ¿cómo te involucraste en esta organización/asociación? ¿Cuánto tiempo llevas en la organización?

2. ¿Qué actividades suelen realizar en la organización/asociación? ¿Todos los miembros participan de la misma manera?

3. En tu caso, ¿en qué actividades sueles participar con mayor frecuencia? ¿En cuáles sueles participar menos? ¿Por qué?

\section{Bloque: Repertorios de Participación Política}

1. En tu experiencia, ¿en qué actividades de participación ciudadana o política suelen participar los jóvenes?

2. ¿En qué tipo de organizaciones los jóvenes suelen participar más?

3. Antes de pertenecer a esta organización/asociación, ¿̇realizabas las mismas actividades de participación? ¿Cómo aprendiste dichas actividades de participación?

4. ¿¿Me puedes mencionar cuáles son las acciones de participación más importantes para ti? ¿Por qué? ¿Qué actividades de participación te agradan más? ¿Por qué?

\section{Bloque: Motivos y Significados de la Participación Política}

1. ¿Qué significa para ti participar en estas actividades?

2. Cuando realizar estas actividades de participación, ¿cómo te sentías? ¿Por qué crees que te sentías así?

3. Me puedes comentar alguna experiencia positiva de participación que haya influido en tu vida

4. ¿Has tenido alguna experiencia desagradable de participación? ¿Cómo fue?

5. ¿Cuáles son los retos que has enfrentado cuando realizabas las actividades de participación (mencionar específicamente cada repertorio y práctica)? ¿Lograste superarlos? ¿Cómo?

6. Recordando tus inicios en la organización/asociación, ¿por qué te involucraste en estas actividades de participación? ¿Y qué te motiva seguir participando?

7. ¿Por qué crees que los jóvenes participan más en dichas temáticas? ¿Por qué crees que otros jóvenes no participan en estas actividades? 
Apéndice D: Análisis factorial de emociones hacia la situación social y política general

Análisis factorial exploratorio con rotación oblicua de las emociones hacia la situación política y social

\begin{tabular}{lcc}
\hline \multicolumn{1}{c}{ Ítems } & $\begin{array}{c}\text { Emociones } \\
\text { negativas }\end{array}$ & $\begin{array}{c}\text { Emociones } \\
\text { positivas }\end{array}$ \\
\hline Indignación & 0.845 & - \\
Ira & 0.820 & - \\
Vergüenza & 0.803 & - \\
\hline Tristeza-Pena & 0.721 & - \\
\hline Optimismo & - & 0.916 \\
Esperanza & - & 0.878 \\
Orgullo & - & 0.741 \\
\hline$\alpha$ de Cronbach por dimensión & .833 & .825 \\
KMO & \multicolumn{2}{c}{.718} \\
Prueba de esfericidad de Bartlett & \multicolumn{2}{c}{$305.01^{* * *}$} \\
Varianza explicada & \multicolumn{2}{c}{$68.31 \%$} \\
\hline$* * *$ p $<.001$ & \multicolumn{3}{c}{} \\
\hline
\end{tabular}

